\title{
Analysing X-ray pulsar profiles
}

\section{Geometry and beam pattern of $4 \mathrm{U} 0115+63$ and V $0332+53$}

\author{
M. Sasaki ${ }^{1}$, D. Müller ${ }^{1}$, U. Kraus ${ }^{2}$, C. Ferrigno ${ }^{3}$, and A. Santangelo ${ }^{1}$ \\ ${ }^{1}$ Institut für Astronomie und Astrophysik, Universität Tübingen, Sand 1, 72076 Tübingen, Germany \\ e-mail: sasaki@astro.uni-tuebingen.de \\ 2 Institut für Physik, Universität Hildesheim, Marienburger Platz 22, 31141 Hildesheim, Germany \\ 3 ISDC Data Centre for Astrophysics, Univeristy of Geneva, Chemin d'Écogia 16, 1290 Versoix, Switzerland
}

Received 13 December 2010 / Accepted 17 January 2012

\begin{abstract}
Aims. By analysing the asymmetric pulse profiles of the transient Be/X-ray binaries $4 \mathrm{U} 0115+63$ and V $0332+53$ we aim to identify the origin of the pulsed emission to understand the geometry of the accretion onto the neutron star.

Methods. We have applied the pulse-profile decomposition method, which enabled us to find two symmetric pulse profiles for the two magnetic poles of the neutron star. We derived beam patterns for different energy bands and luminosity states. This allowed us to identify the components that are responsible for the emission. The analysis and the models used for the interpretation of the results take relativistic light deflection into account.

Results. We find that the magnetic field of the neutron star is distorted in both $4 \mathrm{U} 0115+63$ and V $0332+53$. The beam patterns are interpreted in terms of a model for an accretion column that includes the formation of a halo at the bottom of the accretion column and scattering in the upper accretion stream.

Conclusions. In both systems, an accretion column forms while the accretion rate is high. If the accretion decreases and the sources become fainter, the emission from the halo and the accretion column disappears. In 4U 0115+63 there seems to be significant scattering of photons in the still existing accretion stream even at the end of the outburst. In V 0332+53, the scattering in the upper stream also disappears at the end and we apparently observe the emission from the hot spots on the neutron star.
\end{abstract}

Key words. accretion, accretion disks - stars: neutron - X-rays: binaries

\section{Introduction}

In accreting X-ray pulsars ionised gas from a normal star is accreted onto a neutron star (NS) along the magnetic field lines. This results in the production of X-rays. If the magnetic and rotation axes of the neutron star are misaligned, pulsed emission is observed. These X-ray pulsars exhibit a wide variety of pulse shapes that differ from source to source (White et al. 1983; Frontera \& Dalfiume 1989; Bildsten et al. 1997, and references therein). In addition, the pulse shapes strongly depend on both the energy and the luminosity, as has been observed for Vela X-1 (Staubert et al. 1980), EXO 2030+375 (Parmar et al. 1989), Cen X-3 (Nagase et al. 1992), etc. Efforts have been made for more than three decades to improve our understanding of the physics and the geometries of the accretion and the resulting emission (e.g., Basko \& Sunyaev 1976; Wang \& Welter 1981; Riffert \& Meszaros 1988; Brainerd \& Meszaros 1991; Becker $\&$ Wolff 2007, and references therein). However, owing to the complexity of the problem, there is still no compelling model that can describe the physics of the emission and the observed pulse profiles.

The changes in pulse profiles with luminosity are believed to be related to the change of the structures formed by the accreted gas. In low-luminosity states, the accretion rate is low and the gas can fall freely onto the magnetic poles of the neutron star. It forms a mound on the surface of the neutron star from which a pencil-beam emission is expected (Wang \& Welter 1981). In high-luminosity states, the accretion rate is increased and the gas funneled along the magnetic fields forms an accretion column. A radiative shock forms in the accretion column above the neutron star surface (Davidson 1973; Basko \& Sunyaev 1976), above which the plasma is in free fall. X-rays are radiated from the walls of the accretion column below the shock. A luminous halo can form at the bottom of the column by scattering of photons on the neutron star surface (Davidson \& Ostriker 1973; Lyubarskii \& Syunyaev 1988; Kraus et al. 2003; Ferrigno et al. 2009). The luminosity dependence of the pulse profile thus reflects the transition from low to high accretion rate or vice versa, and hence the change of the emission components. From the observed pulse profiles we are able to derive the beam patterns, i.e., the flux distributions from one emission region, as seen from the distant observer. This again will allow us to derive the local beam pattern as well as the geometry of the neutron star. In doing so it is also necessary to take the relativistic light bending into account.

If the magnetic field is a dipole field and each emission region is axially symmetric with respect to the magnetic axis, the observed pulse profile should be symmetric. However, the observed pulse profiles of X-ray pulsars are in general asymmetric. Different theoretical models have been suggested to explain the observed asymmetry of the pulse profiles. A distorted magnetic dipole field in which the two magnetic poles are not located opposite to each other has been suggested as a possible reason for the asymmetry of pulse profiles (Parmar et al. 1989; Leahy 1991; Riffert et al. 1993; Bulik et al. 1995). Another way to explain the 


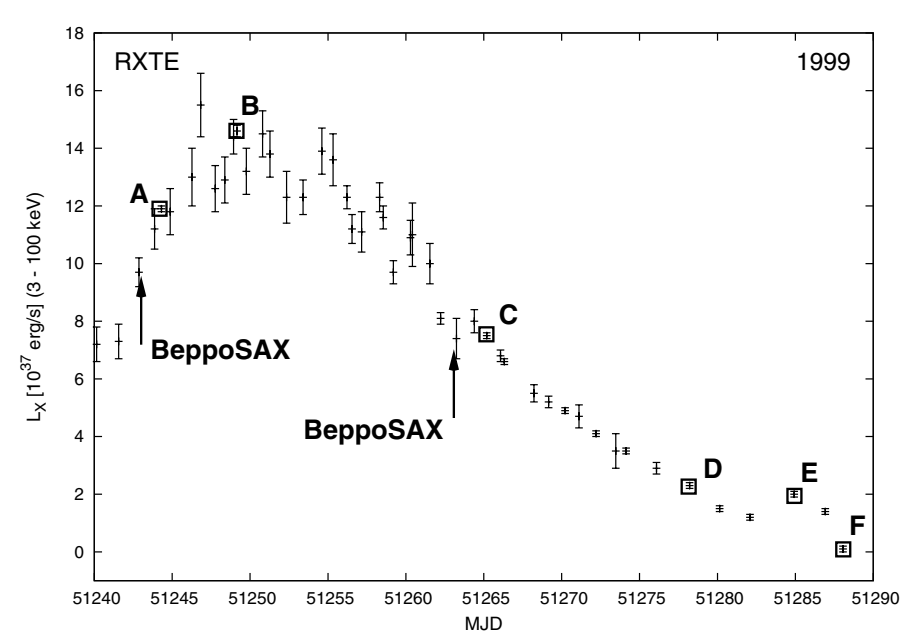

Fig. 1. Light curve obtained from the RXTE observations of 4U 0115+63 during the giant outburst in 1999. Data used in this work are indicated.

asymmetric shape of pulse profiles is an asymmetric accretion stream (Basko \& Sunyaev 1976; Wang \& Welter 1981; Miller 1996), which has also been discussed for other types of accreting neutron stars, e.g., the "Bursting Pulsar" GRO J1744-28, which shows pulse profiles changes during type II bursts caused by instabilities in accretion (e.g., Kouveliotou et al. 1996; Lewin et al. 1996).

Based on the assumption of distorted magnetic fields, Kraus et al. (1995) developed the decomposition method that allows us to find two symmetric contributions from the two poles to the observed total pulse profiles. Using this pulse profile decomposition method, we have successfully analysed the pulse profiles of the X-ray pulsars Cen X-3 (Kraus et al. 1996), Her X-1 (Blum \& Kraus 2000), EXO 2030+375 (Sasaki et al. 2010), and A $0535+26$ (Caballero et al. 2011).

In this paper, we present the results of the pulse profile decomposition analysis of the transient sources $4 \mathrm{U} 0115+63$ and $\mathrm{V} 0332+53$.

\section{Data}

\section{1. $4 U 0115+63$}

4U 0115+63 is an accreting X-ray pulsar, which has been known and was extensively studied since the early 1970s. Observations of the Uhuru satellite yielded detections of this X-ray transient in 1970 and 71 (Forman et al. 1976). The subsequent analysis of the archival data of the Vela 5B satellite revealed that the source had also been detected in 1969 (Whitlock et al. 1989). Rappaport et al. (1978) measured the orbital motion of the source from observations with the SAS 3 satellite in 1978, obtaining an orbital period of $24.3 \mathrm{~d}$, while Cominsky et al. (1978) detected pulsations with a pulse period of $3.61 \mathrm{~s}$. Soon thereafter, a strongly reddened Be-star V635 Cas was found as its optical counterpart (Johns et al. 1978; Hutchings \& Crampton 1981; Kholopov et al. 1981). This star was later classified as a B0.2Ve star at a distance of 7-8 kpc (Negueruela \& Okazaki 2001). Pulse profiles from HEAO-1 observations were presented by Johnston et al. (1978).

In 1999 4U 0115+63 underwent a giant outburst, which is believed to be caused by an enhanced mass loss activity of the Be star. During this outburst the Rossi X-ray Timing Explorer (RXTE) monitored 4U 0115+63 extensively (Fig. 1). The source was also observed by BeppoSAX on March 06 and 26, 1999
Table 1. Data used for the pulse profile decomposition analysis and the luminosities of $4 \mathrm{U} 0115+63$ when the data were obtained.

\begin{tabular}{cccc}
\hline \hline ID & ObsID & MJD & $L_{3-100 \mathrm{keV}}$ \\
\hline & 006534 & 51243 & $9.4 \times 10^{37} \mathrm{erg} \mathrm{s}^{-1}$ \\
$\mathrm{~A}$ & $40070-01-01-00$ & 51244 & $1.2 \times 10^{38} \mathrm{erg} \mathrm{s}^{-1}$ \\
$\mathrm{~B}$ & $40070-01-03-00$ & 51249 & $1.5 \times 10^{38} \mathrm{erg} \mathrm{s}^{-1}$ \\
& 006631 & 51263 & $6.3 \times 10^{37} \mathrm{erg} \mathrm{s}^{-1}$ \\
$\mathrm{C}$ & $40070-01-04-00$ & 51265 & $7.5 \times 10^{37} \mathrm{erg} \mathrm{s}^{-1}$ \\
$\mathrm{D}$ & $40051-05-12-00$ & 51278 & $2.3 \times 10^{37} \mathrm{erg} \mathrm{s}^{-1}$ \\
$\mathrm{E}$ & $40411-01-24-00$ & 51285 & $2.0 \times 10^{37} \mathrm{erg} \mathrm{s}^{-1}$ \\
$\mathrm{~F}$ & $40411-01-26-00$ & 51288 & $0.1 \times 10^{37} \mathrm{erg} \mathrm{s}^{-1}$ \\
\hline
\end{tabular}

Notes. Data with ID A - F were taken by RXTE, the first and the fourth data-sets are from BeppoSAX. The luminosity was calculated assuming a distance of $7 \mathrm{kpc}$.

before and after the maximum. Observations with BeppoSAX revealed that the spectrum of $4 \mathrm{U} 0115+63$ shows four cyclotron resonance scattering features (CRSFs) at 12.7, 24.2, 35.7, and $49.5 \mathrm{keV}$ (Santangelo et al. 1999), which was the first discovery of multiple absorption lines due to cyclotron resonant scattering ever. Additional analyses of the RXTE and BeppoSAX data of the giant outburst of 4U 0115+63 in 1999 have been published by Nakajima et al. (2006), Tsygankov et al. (2007), Ferrigno et al. (2009), and Baushev (2009).

The pulse profiles of $4 \mathrm{U} 0115+63$ and also other accreting $\mathrm{X}$-ray pulsars are strongly luminosity- and energy-dependent. Because we aim to understand the reason for the evolution of the pulse shapes with luminosity and energy, it is crucial to have statistically useful pulse profiles of the source in different luminosity states, hence different observations during an outburst, as well as for different energy bands. Moreover, the more pulse profiles we have, the better will be the determination of the symmetry points and thus the determination of the geometry parameters of the neutron star (see Sect. 3).

We used the data of six RXTE observations taken with the Proportional Counter Array (PCA, Jahoda et al. 1996) and two BeppoSAX observations (Ferrigno et al. 2009). For the BeppoSAX data, we used the same energy bands as in Ferrigno et al. (2009) and divided the RXTE data into similar bands. The selected bands are approximately $<5 \mathrm{keV}, 5-10 \mathrm{keV}, 10-15 \mathrm{keV}$, 15-20 keV, 20-30 keV, and $>30 \mathrm{keV}$. If the statistics were not good enough (observations D, E, and F), we combined the harder energy bands, e.g., creating pulse profiles for the band 15-115 keV. For the same reason, we did not use the hardest band $(>15 \mathrm{keV})$ in the last and thus faintest observation $\mathrm{F}$ for the pulse profile analysis. Table 1 lists the data used in our work. All used pulse profiles are shown in Figs. 2 and 3.

First, the light curves were corrected for barycentric and orbital motions and folded with pulse periods of $\sim 3.61 \mathrm{~s}$ derived for each observation. The light curves were binned into 128 phase bins in most cases. Only for observations $\mathrm{E}$ and $\mathrm{F}$ we created pulse profiles with 64 phase bins because of poor statistics. In the next step, ideally, the pulse profiles should be phase-connected. However, the high degree of timing noise and the sparse nature of the observations prevented us from doing this. Therefore, the pulse profiles were then shifted by using their common morphological properties. In particular, we focused on the two sharp minima immediately before the major peak and used them as reference points. These minima are seen at phase 0.0 and 0.1 in Figs. 2 and 3, best visible in the softest band (top row) and in the $10-15 \mathrm{keV}$ band data of the observation $\mathrm{F}$. All profiles were then normalised to their maximum 


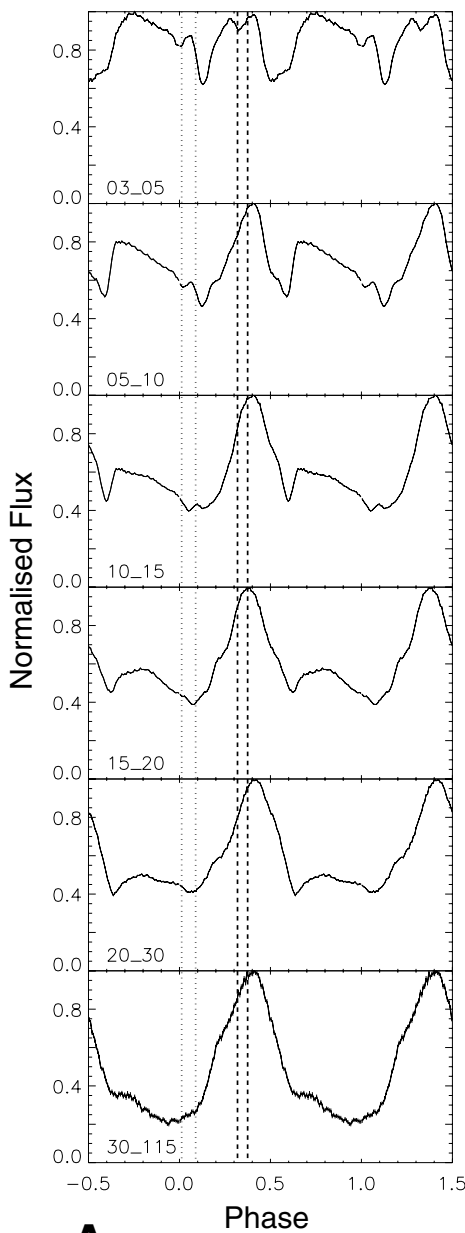

A

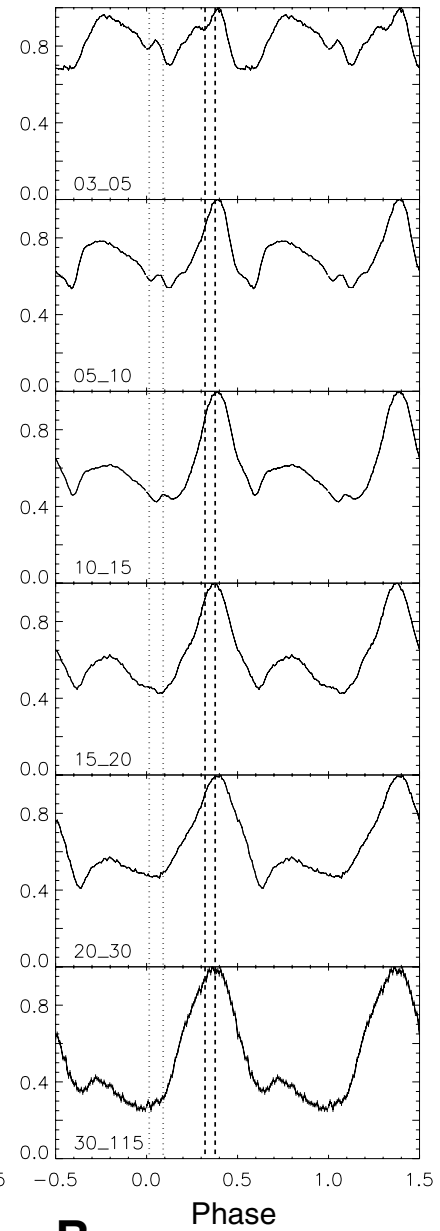

B

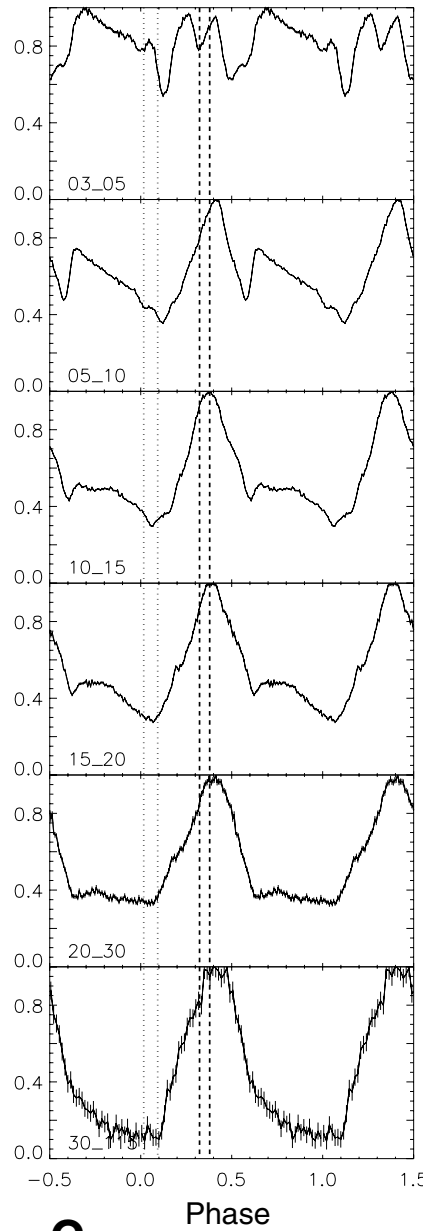

C

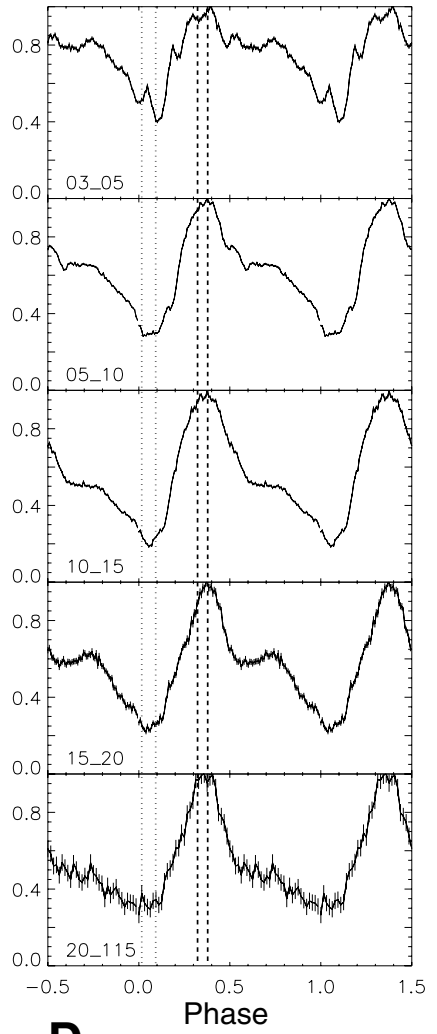

D

Fig. 2. Pulse profiles of 4 U $0115+63$ during the giant outburst in 1999 observed with RXTE for the observations A-D. Energy bands are soft to hard from top to bottom for each observation. The numbers in the plots indicate the bands (lower energy - upper energy in keV). The dotted and dashed lines show the ranges in which the symmetry points $\Phi_{1}, \Phi_{2}$ are found (see Sect. 3).

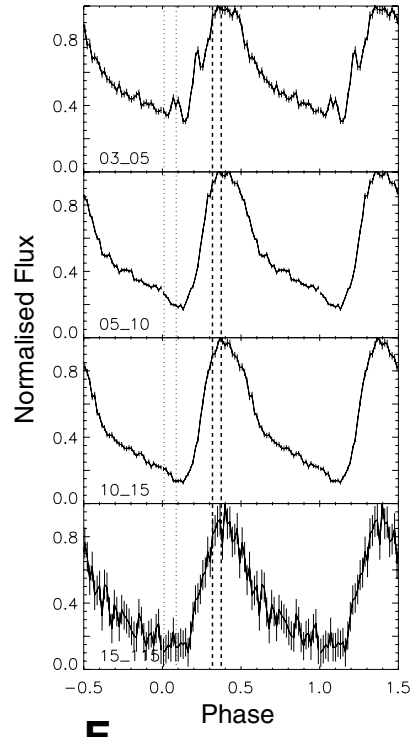

E

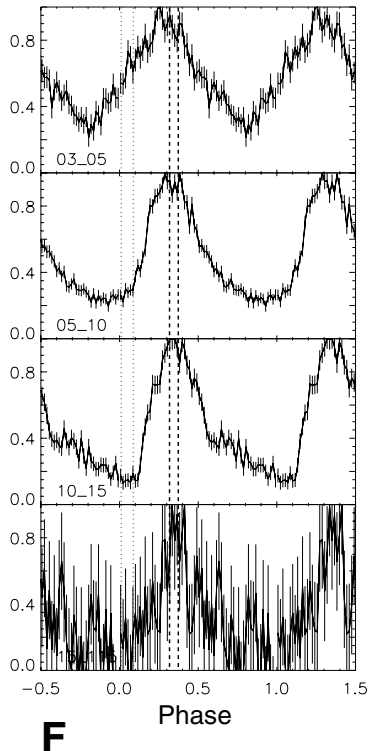

$\mathbf{F}$

For energies higher than $\sim 5 \mathrm{keV}$, the pulse profiles have a major maximum at phase $=0.4$ and an additional hump that follows the maximum. This hump is stronger for lower energies and is visible only during the giant outburst (observations A-D). It seems that it has disappeared in the data-sets $\mathrm{E}$ and $\mathrm{F}$ at the end of the outburst.

The most intriguing band is the softest band below $5 \mathrm{keV}$. The major peak has an additional component at phase $=0.25$ in the data near the maximum of the giant outburst (A, B, C, see also Fig. 1 in Ferrigno et al. 2009). Furthermore, the height of the hump at phase $=0.6-1.1$ is comparable to the double peak at 0.4 . The hump and the additional peak seem to be caused by the change of emission characteristics due to the increased accretion during the giant outburst.

For the subsequent analysis we used the following identifier for the pulse profiles with the format LNNNN. The letter $\mathrm{L}$ is the ID for the observations as given in Table 1. The next two following numbers indicate the lower energy of the energy band, the last two numbers for the upper energy. For example, A0305 is the beam pattern belonging to the pulse profile from the A (40070-01-01-00) data for the energy band of 3 to $5 \mathrm{keV}$. The number 11 for the upper energy in the highest energy bands stands for $115 \mathrm{keV}$. For the BeppoSAX data, we used the identifiers " $b$ " for the observation before the maximum and "s" for after maximum.

value. Using fairly broad energy bands limits the impact of the different energy resolution and therefore the combined data-set of different instruments can be safely used in the analysis. 


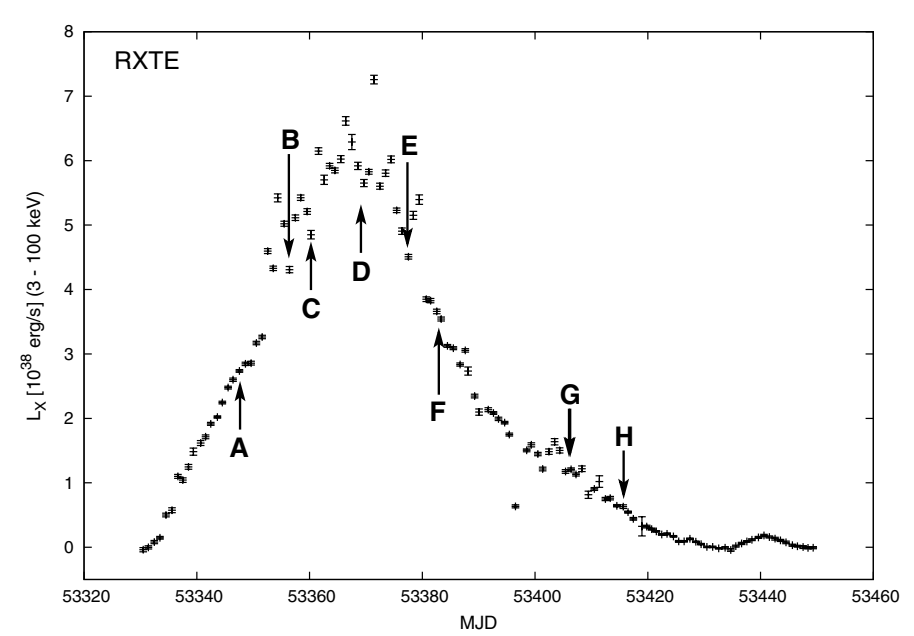

Fig. 4. Light curve of V $0332+53$ during the 2004/2005 outburst obtained with the All-Sky Monitor on RXTE. The selected observations for the pulse profile decomposition are marked.

\section{2. $\vee 0332+53$}

V $0332+53$ was discovered in 1973 by the Vela 5B satellite during a type II, i.e., a giant outburst (Terrell \& Priedhorsky 1984). The pulse period was determined to a value of $4.4 \mathrm{~s}$, while an orbital period of $34 \mathrm{~d}$ was measured (Stella et al. 1985). Its optical counterpart was identified as the O8-9Ve star BQ Cam (Bernacca et al. 1984; Negueruela et al. 1999) with a distance of $\sim 7 \mathrm{kpc}$. Ten years later, there was a second outburst, this time of type I corresponding to a periastron passage of the neutron star, detected with the Tenma satellite (Inoue 1985). The source became again active in 1989 in form of a type II outburst, and the next outburst of type II was measured at the end of 2004 (December 2004 to February 2005, Zhang et al. 2005; Mowlavi et al. 2006, see Fig. 4). About a year before each outburst, one could observe a brightening of the optical companion that was most likely triggered by a sudden increase of the mass ejection rate from the companion star (Mowlavi et al. 2006). Similar to $4 \mathrm{U} 0115+63$, this source is one of the few sources that show multiple cyclotron lines in their spectra. V $0332+53$ is the second X-ray accreting pulsar that has at least three CRSFs, the main harmonic at $E_{\mathrm{cycl}, 1}=25 \mathrm{keV}$ and the first and second harmonics at $E_{\mathrm{cycl}, 2}=51 \mathrm{keV}$ and $E_{\mathrm{cycl}, 3}=72 \mathrm{keV}$, respectively (Coburn et al. 2005; Kreykenbohm et al. 2005; Pottschmidt et al. 2005).

The pulse profiles of $\mathrm{V} 0332+53$ are highly asymmetric and lumimosity-dependent (Zhang et al. 2005; Tsygankov et al. 2006, see Fig.5). One can observe a change in the structure of the profiles from double-peaked to single-peaked and also a change of the phase separation of the double pulses with the source luminosity. While the source was bright, the separation of the two main peaks in the pulse profile remained nearly constant at a value of about 0.47 and decreased to 0.37 within a short time of about three days during which the luminosity declined after the maximum intensity was reached (Zhang et al. 2005). Significant changes of the double-peaked structure of the pulse profiles can be seen as the intensity of the source decreases. For a luminosity of about $L \approx 7 \times$ $10^{37} \mathrm{erg} \mathrm{s}^{-1}$ the profiles become asymmetrically single-peaked at energies below the energy of the main harmonic cyclotron line, while it seems to remain double-peaked above this energy
Table 2. Data used for the pulse profile decomposition analysis of V $0332+53$

\begin{tabular}{cccc}
\hline \hline ID & Observation ID & MJD & $L_{3-100 \mathrm{keV}}$ \\
\hline $\mathrm{A}$ & $90089-11-02-08$ & 53346 & $2.6 \times 10^{38} \mathrm{erg} \mathrm{s}^{-1}$ \\
$\mathrm{~B}$ & $90089-11-03-04$ & 53353 & $4.1 \times 10^{38} \mathrm{erg} \mathrm{s}^{-1}$ \\
$\mathrm{C}$ & $90089-11-04-03$ & 53358 & $4.6 \times 10^{38} \mathrm{erg} \mathrm{s}^{-1}$ \\
$\mathrm{D}$ & $90427-01-01-01$ & 53368 & $4.8 \times 10^{38} \mathrm{erg} \mathrm{s}^{-1}$ \\
$\mathrm{E}$ & $90427-01-02-02$ & 53376 & $3.9 \times 10^{38} \mathrm{erg} \mathrm{s}^{-1}$ \\
$\mathrm{~F}$ & $90014-01-01-04$ & 53381 & $3.4 \times 10^{38} \mathrm{erg} \mathrm{s}^{-1}$ \\
$\mathrm{G}$ & $90014-01-05-01$ & 53407 & $1.1 \times 10^{38} \mathrm{erg} \mathrm{s}^{-1}$ \\
$\mathrm{H}$ & $90427-01-04-04$ & 53414 & $7.9 \times 10^{37} \mathrm{erg} \mathrm{s}^{-1}$ \\
\hline
\end{tabular}

Notes. The luminosity was calculated assuming a distance of $7 \mathrm{kpc}$.

(Tsygankov et al. 2006). However, the low statistics make detailed analyses at these low luminosities difficult.

For the pulse profile decomposition we selected a set of RXTE observations covering the whole time span of the 2004/2005 outburst from the beginning of December 2004 until mid February 2005. These observations are listed in Table 2. The selected observations are also marked in the light curve of the 2004/2005 outburst taken by the RXTE All-Sky Monitor (ASM, see Fig. 4). The data are mainly available as binned-mode data. Therefore, after the event arrival time was corrected for barycentric and orbital motions, pulse profiles were created with phase bins of 128 for four different energy bands: 3-7 keV, 7-20 keV, 20-30 keV, and 30-115 keV. As was discussed before, the pulse profiles of $\mathrm{V} 0332+53$ have two distinct minima that become stronger if the source becomes brighter. In addition, the two maxima have different shapes, one showing a slightly increasing plateau (at phase $0.1-0.4$ in Fig. 5) while the other is flat or has a decreasing plateau (at phase 0.6-0.9). By identifying these two minima and two maxima, we shifted the pulse profiles by defining the minimum before the maximum that shows an increase as phase 0 . For the pulse profiles of $\mathrm{V} 0332+53$ we used the same scheme for the identifiers as for $4 \mathrm{U} 0115+63$ : the ID given in Table 2 together with the lower and upper energies gives the total identifier, e.g., the pulse profile of the softest band of the observation 90089-11-02-08 is called A0307.

\section{The analysis}

A detailed description of the pulse profile decomposition method can be found in Kraus et al. (1995). A short summary of the method is also given in our papers on EXO 2030+375 (Sasaki et al. 2010) and A 0535+26 (Caballero et al. 2011). In Fig. 6 we show the assumed geometry of the neutron star and the angles used for the analysis. The polar angles of the two magnetic poles are $\Theta_{1}$ and $\Theta_{2}$. We assumed that there is an offset angle $\delta$ between the second magnetic pole and the antipodal position of the first magnetic pole. The inclination angle of the observer with respect to the rotation axis of the neutron star is $\Theta_{0}$. The angle between the line of sight and the axis through one magnetic pole is called $\theta_{\text {obs }}$. As the neutron star rotates, $\theta_{\text {obs }}$ changes with the rotation angle $\Phi$. Since the observed X-ray emission most likely arises from regions close to the magnetic poles as well as directly above, and we assumed that the emission is symmetric in shape, there should be two symmetry points in the emission of each magnetic pole at $\Phi_{i}$ and $\Phi_{i}+\pi(i=1,2)$, each corresponding to the pulse phase at which the pole is either closest or farthest from the line of sight. The non-antipodal positions of the two 


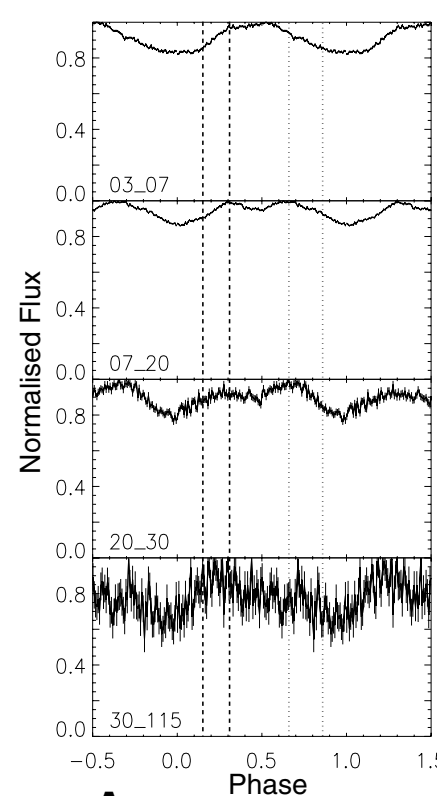

A

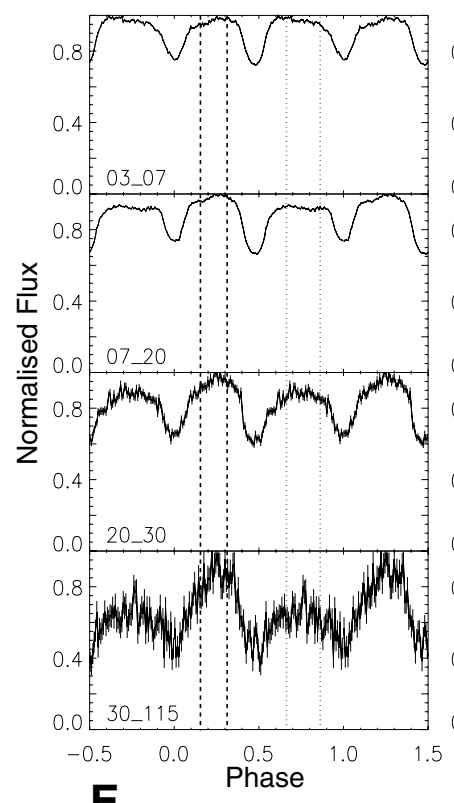

$\mathbf{E}$

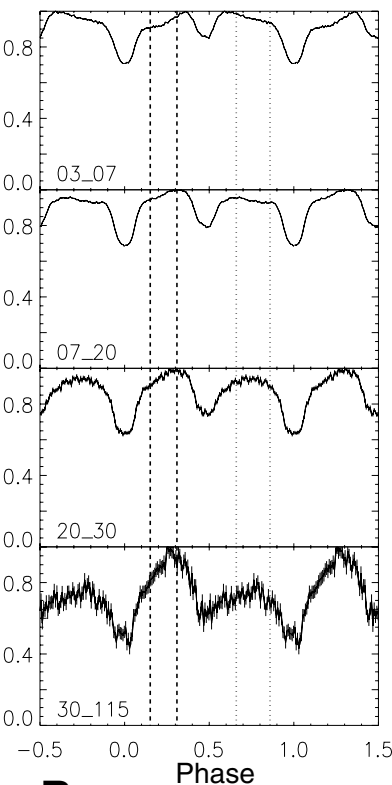

B

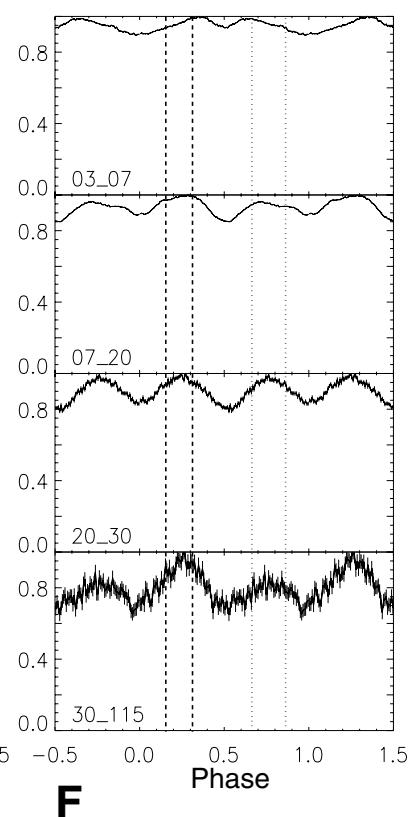

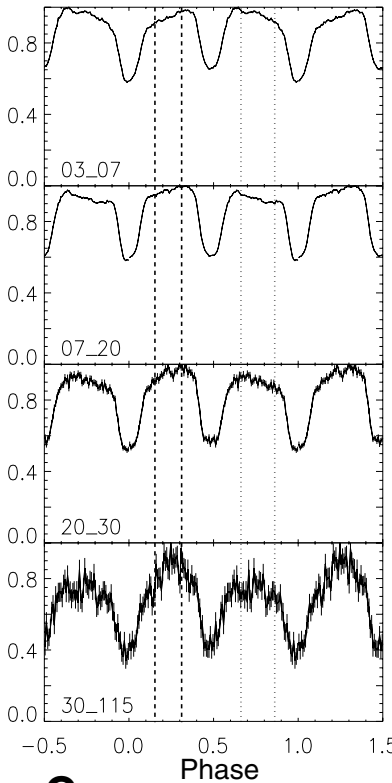

C

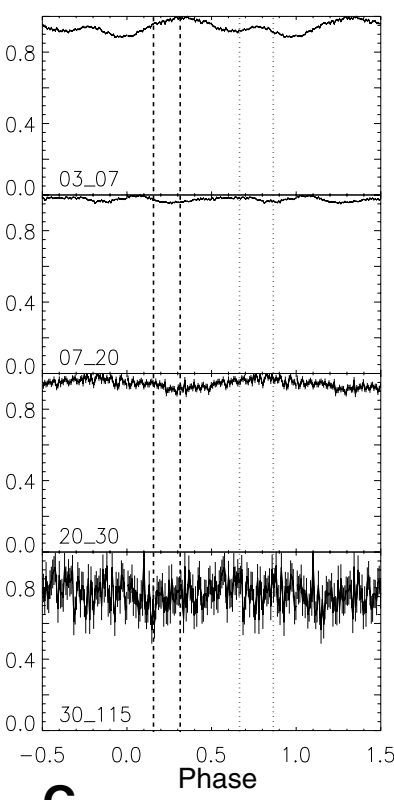

G

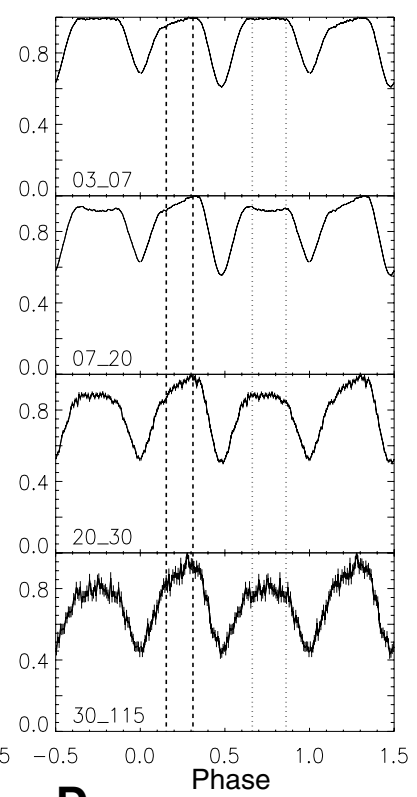

D

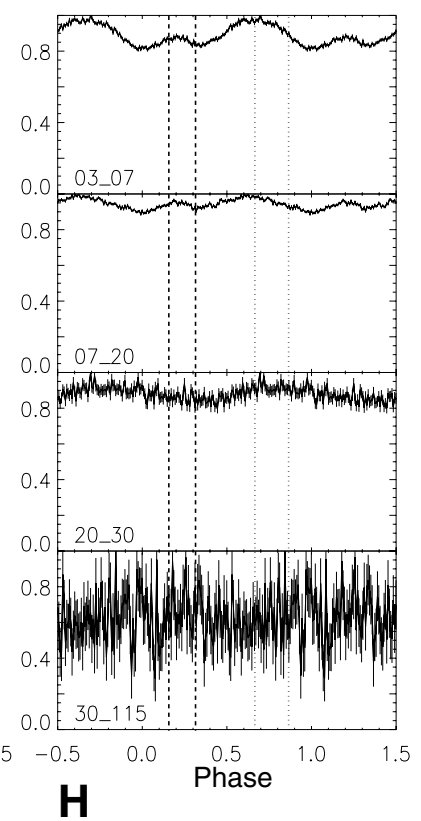

Fig. 5. Pulse profiles of V 0332+53 during the giant outburst in 2004/2005 observed with RXTE. The pulse profiles are sorted chronologically from left to right in the upper panel, then left to right in the lower panel. Energy bands are soft to hard from top to bottom for each observation. The bands (lower energy - upper energy in $\mathrm{keV}$ ) are given in the diagrams. The letters at the botton of each panel give our identifiers for the observations. The dotted and dashed lines show the ranges in which the symmetry points $\Phi_{1}, \Phi_{2}$ are found (see Sect. 3).

poles result in an offset $\Delta=\pi-\left(\Phi_{1}-\Phi_{2}\right)$ in rotation angle between the symmetry points $\Phi_{1}$ and $\Phi_{2}$.

The decomposition method is in principle a backwardtracing of the emission. The basic assumption is that the two magnetic poles of the neutron star have the same emission characteristics and that a sum of the two symmetric single-pole pulse profiles result in the observed asymmetric pulse profile. We are interested in the shape and the change of the shape of the beam patterns with energy and luminosity. This gives information about the relative fluxes of the different emission regions of the source (see Sect. 4) that contribute to the observed total emission. Therefore, the observed total pulse profiles are first normalised so that the maximum in the pulse profile is equal to one.
To decompose the observed asymmetric pulse profiles into two symmetric pulse profiles, each of these normalised asymmetric pulse profiles $F$ can be represented by its Fourier expansion:

$$
F(\Phi)=\frac{1}{2} u_{0}+\sum_{k=1}^{n / 2-1}\left[u_{k} \cos (k \Phi)+v_{k} \sin (k \Phi)\right]+u_{n / 2} \cos \left(\frac{n}{2} \Phi\right)
$$

where $n$ is the number of phase bins, which we divide the pulse profiles into (64 or 128, depending on the strength of the signal in each observation). As shown by Kraus et al. (1995), we can express $F(\Phi)$ by means of two symmetric functions $f_{1}$ and $f_{2}$ for 
A\&A 540, A35 (2012)

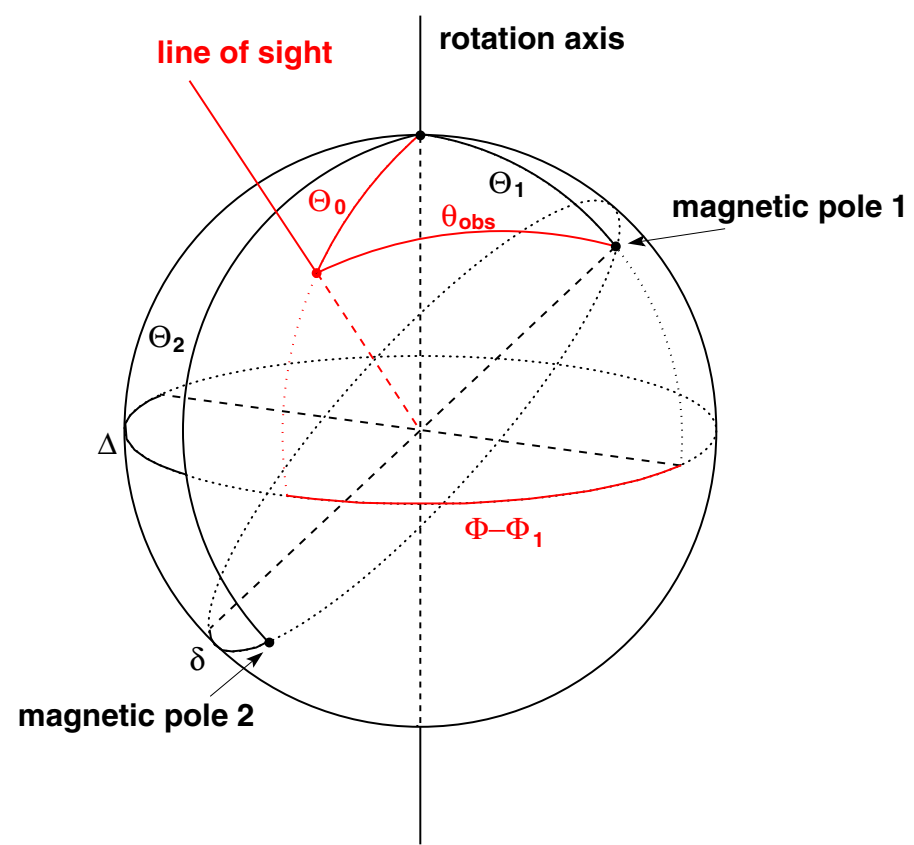

Fig. 6. Intrinsic geometry of the neutron star.

virtually any choice of two symmetry points $\Phi_{1}$ and $\Phi_{2}$. Because $f_{1}$ and $f_{2}$ should be symmetric, the sine terms vanish and we can write

$f_{1}(\Phi)=\frac{1}{2} c_{0}+\sum_{k=1}^{n / 2} c_{k} \cos \left[k\left(\Phi-\Phi_{1}\right)\right]$,

$f_{2}(\Phi)=\frac{1}{2} d_{0}+\sum_{k=1}^{n / 2} d_{k} \cos \left\{k\left[\Phi-\left(\Phi_{2}-\pi\right)\right]\right\}$.

The parameters $c_{0}$ and $d_{0}$ are subject to the constraint that their sum equals $u_{0}$. They are determined in two steps: in the decomposition, minimum values $c_{0, \min }$ and $d_{0 \text {,min }}$ are determined such that the symmetric functions do not become negative at any phase. The remaining constant flux $u_{0}-c_{0, \min }-d_{0, \min }$ is divided up when the two beam patterns are combined to obtain the total beam pattern (as described in Sect. 3.1.1). It is distributed among the two beam patterns so that the quadratic deviation in the overlap region is minimised. The mean square devitaion function $Q(a, b)$ is computed with the optimum flux distribution for each pair of values $a$ and $b$ (see Sect.3.1.1). We assumed that $F$ is well-defined by the $n$ data points and is thus limited to frequencies below $n / 2$. This is confirmed by verifying that the power of the Fourier transform of $F$ approaches zero if the frequency approaches $n / 2$. As sugested by Kraus et al. (1995), we used less than $n$ Fourier coefficients, omitting the highest coefficients that may be affected by aliasing and may also have fairly large statistical errors. This results in a certain smoothing of the pulse profile. In practice, we performed the decomposition with 20 to 30 Fourier coefficients. In addition, we used $\Delta=\pi-\left(\Phi_{1}-\Phi_{2}\right)$ for convenience instead of $\Phi_{2}$, i.e., searching for parameters $\left(\Phi_{1}, \Delta\right)$.

For each pulse profile, we then selected the symmetry points that yield a "good" decomposition according to the following criteria.

1. Positive flux: the Fourier analysis yields two symmetric functions $f_{1}$ and $f_{2}$ for each pulse profile. These functions must be positive because they represent the emitted flux of an astronomical object.
Table 3. Parameters obtained from the decomposition analysis of the pulse profiles of $4 \mathrm{U} 0115+63$ and $\mathrm{V} 0332+53$.

\begin{tabular}{|c|c|c|}
\hline & $4 \mathrm{U} 0115+63$ & $\mathrm{~V} 0332+53$ \\
\hline \multicolumn{3}{|c|}{ Decomposition parameters } \\
\hline$\Phi_{1}$ & $115^{\circ}-135^{\circ}$ & $91^{\circ}-110^{\circ}$ \\
\hline$\Phi_{2}$ & $5^{\circ}-30^{\circ}$ & $277^{\circ}-311^{\circ}$ \\
\hline$\Delta$ & $60^{\circ}-75^{\circ}$ & $6^{\circ}-21^{\circ}$ \\
\hline$a$ & $-1.2(+1.0,-1.6)$ & $-0.95(+0.35,-1.05)$ \\
\hline$b$ & $1.8(+0.2,-1.3)$ & $1.0(+0.5,-0.5)$ \\
\hline \multicolumn{3}{|c|}{ Pulsar geometry } \\
\hline$\Theta_{1}$ & $148^{\circ}\left(+12^{\circ},-57^{\circ}\right)$ & $142^{\circ}\left(+18^{\circ},-22^{\circ}\right)$ \\
\hline$\Theta_{2}$ & $74^{\circ}\left(+31^{\circ},-44^{\circ}\right)$ & $38^{\circ}\left(+8^{\circ},-12^{\circ}\right)$ \\
\hline$\delta$ & $65^{\circ}\left(+14^{\circ},-26^{\circ}\right)$ & $12^{\circ}\left(+24^{\circ},-1^{\circ}\right)$ \\
\hline$\Theta_{0}$ (assumed) & $60^{\circ}$ & $70^{\circ}$ \\
\hline
\end{tabular}

Notes. The ranges in which decompositions were found to be consistent for all analysed observations and energy bands are given for $\Phi_{1}, \Phi_{2}$, and $\Delta$. The main uncertainty comes from the poorly known or unknown value of $\Theta_{0}$.

2. No ripples: the functions $f_{1}$ and $f_{2}$ describe the emission of two poles that emit independently from each other, therefore, one expects that they do not show features that match exactly. Also, we do not expect the single-pole pulse profiles to be more complex than the observed total pulse profiles. Therefore, the two functions are not supposed to have smallscale fluctuations that cancel out in the sum.

3. Same geometry: we used several pulse profiles from different observations and energy ranges. It is crucial to find a small region in the parameter space that is allowed for all cases, because the emission arises from only one object that has one special geometry. Therefore, the symmetry points must be the same for the decompositions of all available data.

For each observed pulse profile, $\Phi_{1}$ and $\Delta$ (and thus $\Phi_{2}$ ) were determined independently. The comparison of the possible solutions in the parameter space of $\Phi_{1}$ and $\Delta$ for different energy bands and different observations, thus luminosity states, then yields a few, or, ideally only one region in the $\Phi_{1}-\Delta$ parameter space with pairs of $\Phi_{1}$ and $\Delta$, which have similar values for all pulse profiles. These $\Phi_{1}$ and $\Delta$ values were used for the subsequent analysis for each pulse profile. By means of the resulting two symmetry points, we reconstructed the single-pole pulse profiles for each observed pulse profile.

Each single-pole pulse profile obtained in the decomposition has two symmetry points that correspond to the rotation angles at which the pole is closest to, or farthest from, the line of sight, respectively. Since there is no way to decide which symmetry point corresponds to which end of the range in viewing angle, two groups of solutions are obtained that we call plus and minus solutions. From these single-pole pulse profiles of the two groups of solutions we derived the beam patterns and interpreted them in terms of emission originating from the accretion column, halo, and/or the upper accretion stream. Comparisons of the beam patterns to theoretically calculated models for the emission from an accreting neutron star (e.g., Kraus et al. 2003, in prep., see Sect.4) will tell us which of these two groups of solutions are more reasonable.

We summarised the parameters that we obtained from the decomposition analysis and those derived for the geometry of the neutron star for $4 \mathrm{U} 0115+63$ and V $0332+53$ in Table 3 . 


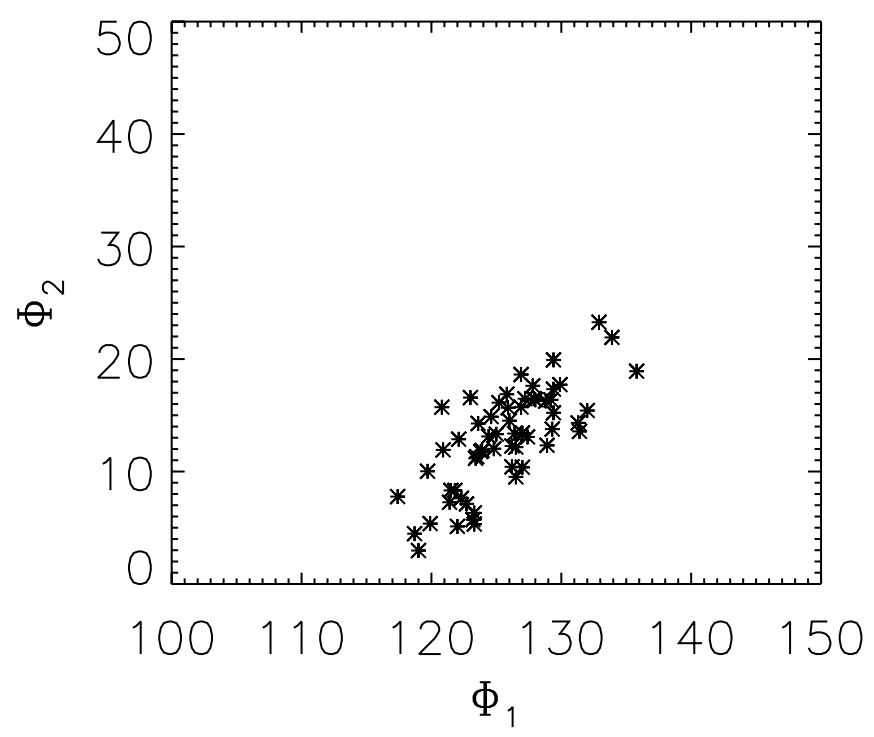

Fig. 7. Diagram showing the values of $\Phi_{1}$ and $\Phi_{2}$ for each pulse profile for the solution of $4 \mathrm{U} 0115+63$.
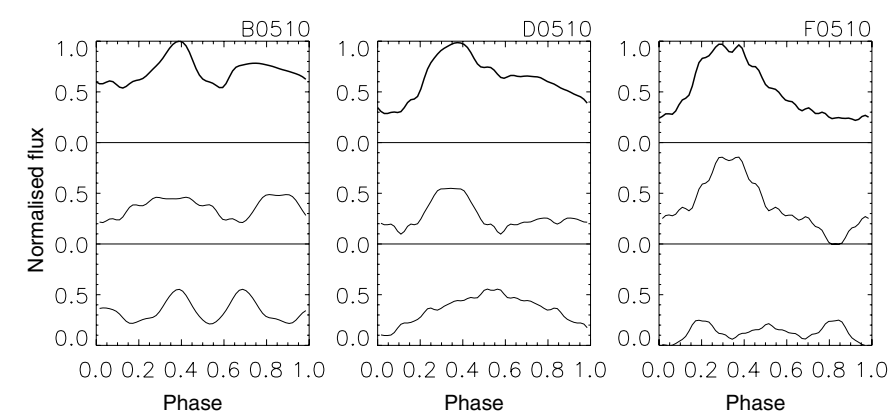

Fig. 8. Single-pole pulse profiles of $4 \mathrm{U} 0115+63$ for one pole (lower curve) and the other (middle) for the observations B (maximum), D (decay), F (end of decay) in the 5-10 keV band. The upper thick curve shows the total pulse profile, which is the sum of the two single-pole pulse profiles.

\section{1. $4 \cup 0115+63$}

The decomposition of all pulse profiles has yielded one set of solutions that can be regarded as consistent for all observed pulse profiles: the first symmetry point is found in the range of $\Phi_{1}=115^{\circ}-135^{\circ}$ and the offset of the second symmetry point from $\Phi_{1}+\pi$ (i.e., an antipodal configuration) has the range of $\Delta=60^{\circ}-75^{\circ}$. A diagram with the obtained values for $\Phi_{1}$ and $\Phi_{2}$ for each pulse profile is shown in Fig. 7. Figure 8 shows an example of the two single-pole pulse profiles obtained from the Fourier decomposition (middle and bottom rows) and their sum, which is the total pulse profile (top). We can derive the geometric parameters $\Theta_{1}, \Theta_{2}$, and $\delta$ from this set of solutions, with $\Theta_{1,2}$ being the polar angles of the magnetic poles with respect to the rotation axis and $\delta$ being the offset of the second magnetic pole from the antipodal position of the first magnetic pole. To obtain these geometrical parmeters of the neutron star and also for the reconstruction of the beam patterns, we need to know the inclination angle $\Theta_{0}$ of the observer with respect to the rotation axis of the neutron star. However, this number is not known for most of the accreting X-ray pulsars. For 4U 0115+63, Negueruela \& Okazaki (2001) have derived a companion mass of $\sim 18 M_{\odot}$ from the optical spectrum, corresponding to an inclination angle of the binary system $i \approx 40-60^{\circ}$. We assumed that the rotation axis of the neutron star is perpendicular to the orbital plane, i.e., $\Theta_{0} \approx i$. For the pulse profile analysis, we will assume $\Theta_{0}=60^{\circ}$.

\subsubsection{Asymptotic beam patterns}

From the single-pole pulse profiles obtained by decomposing the observed pulse profiles, we can calculate the beam patterns for each pole as seen by the distant observer. One of our basic assumptions was that the two poles of the NS have the same beam patterns. Therefore, while the NS rotates, we see emission from pole 1 at phase $\Phi$ and the angle $\theta_{\text {obs }}$ to pole 1 , which may be the same as the emission from pole 2 at the same angle $\theta_{\text {obs }}$ to pole 2 but at a different phase $\tilde{\Phi}$. For pole 1 the angle $\theta_{\text {obs }}$ varies between $\theta_{\text {obs,min }}=\left|\Theta_{1}-\Theta_{0}\right|$ and $\theta_{\text {obs, } \max }=\Theta_{1}+\Theta_{0}$ over the entire range of $\Phi / 2 \pi=0-1$ (see Fig. 6). The pulse profile of pole 1 therefore displays the section of the beam pattern between $\theta_{\text {obs,min }}$ and $\theta_{\text {obs,max }}$. The same holds for pole 2 from which we see a different fraction of the beam pattern, which however might be partly identical with the visible fraction of the beam pattern of pole 1. If we find such identical parts of the two poles, we can overlay and connect the beam patterns and derive the relation between $\cos \left(\Phi-\Phi_{1}\right)$ and $\cos \left(\tilde{\Phi}-\Phi_{2}\right)$

$\cos \left(\Phi-\Phi_{1}\right)=a+b \cos \left(\tilde{\Phi}-\Phi_{2}\right), \quad b>0$,

by determining the parameters a and b (see Kraus et al. 1995, for details).

This step of the analysis is based on the assumption that the two emission regions have identical beam patterns. There might also be some cases for which this is incorrect. However, in such cases we should see an inconsistency between the two beam patterns derived from the two single-pole pulse profiles and it will not be possible to reconstruct the total asymptotic beam pattern from the decomposition. If we obtain two parts of beam patterns that can be connected, we believe that the assumption of identical beam patterns is viable.

We shifted and scaled the two beam patterns computed from the decomposed pulse profiles as shown in Fig. 8 to obtain the best match of the two parts for a certain range of $\theta_{\text {obs }}$ for all observations and all energy bands. For different values of the parameters $a$ and $b$ we computed the mean square deviation $Q$ between the two beam patterns in the overlapping part. The minimum of the function $Q(a, b)$ was determined to obtain $a$ and $b$ for the best overlap for all observations and energy bands. The scattering of $a$ and $b$ obtained for each data-set represents the uncertainties of these parameters. For the pulse profiles of $4 \mathrm{U} 0115+63$, we obtained $a=-1.2(+1.0,-1.6)$, $b=1.8(+0.2,-1.3)$. The reconstructed asymptotic beam patterns seen by the distant observer are shown in Fig. 9.

The asymptotic beam patterns in Fig. 9 are plotted over the angle $\theta_{\text {obs }}$ between the normal at the first magnetic pole and the line of sight. The difference between the plus and minus solutions is the assignment of the angle $\theta_{\mathrm{obs}}$ : replacing $\theta_{\mathrm{obs}}$ with $\pi-\theta_{\text {obs }}$ transforms the beam patterns of the plus solution into those of the minus solution, and vice versa. Therefore, the features that are seen in the plus solution for, e.g., lower $\theta_{\text {obs }}$ are found for higher $\theta_{\mathrm{obs}}$ in the minus solution. The most prominent features in the beam patterns are the maximum for lower $\theta_{\text {obs }}$ and the steep rise for higher $\theta_{\mathrm{obs}}$ for the minus solution (or the steep rise in flux for lower $\theta_{\text {obs }}$ and the maximum seen in the softer bands for higher $\theta_{\text {obs }}$ for the plus solution ). In a system where a large amount of matter is accreted and the major part of the emission is caused by scattering, it is unlikely to obtain dominant flux for low $\theta_{\text {obs }}$ (i.e., a pencil beam) for this wide range 

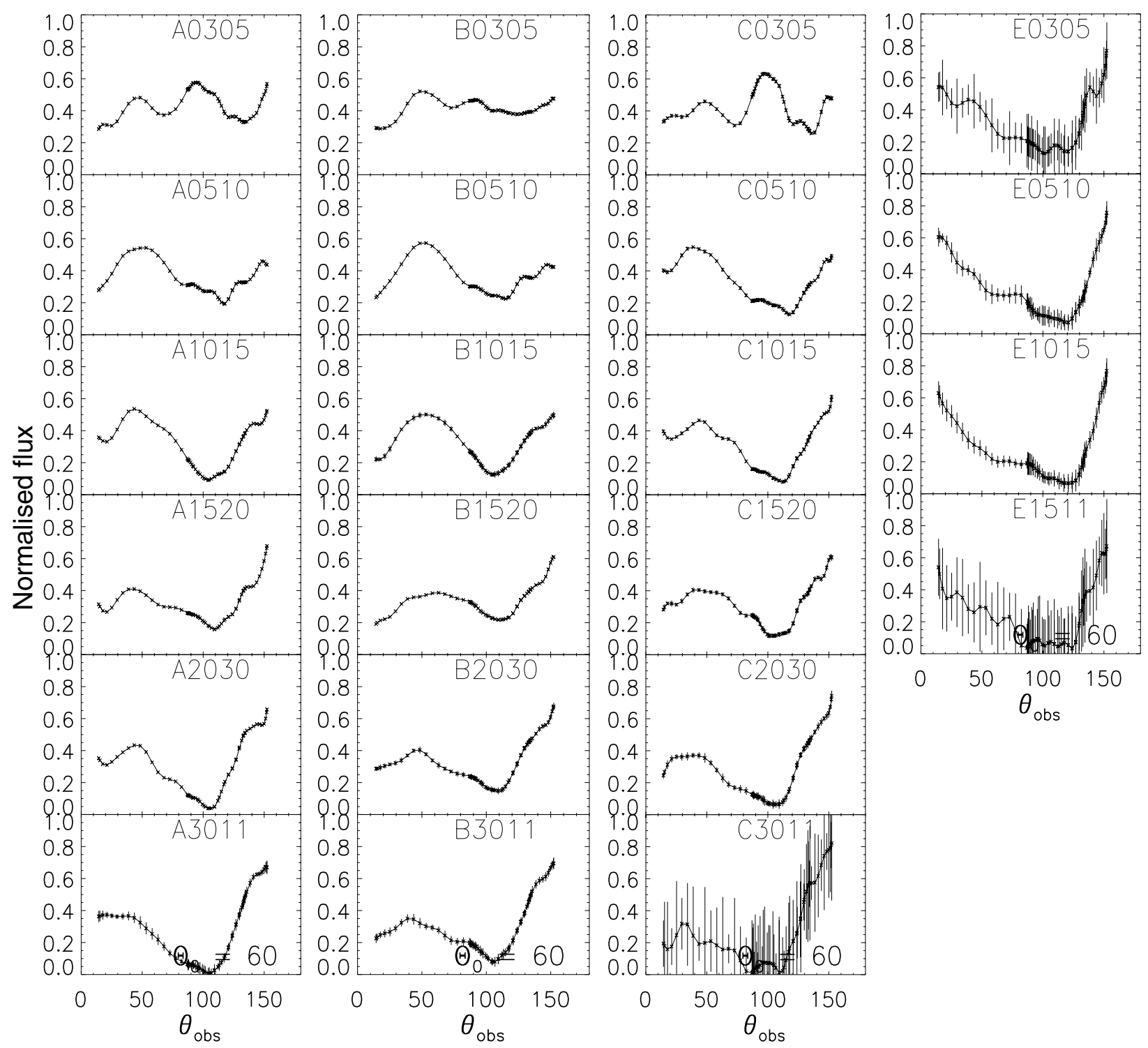

Fig. 9. Asymptotic beam patterns of $4 \mathrm{U} 0115+63$ as seen by the distant observer plotted over the angle $\theta_{\mathrm{obs}}$ between the first magnetic pole and the line of sight for the observations A (before maximum), B (maximum), C (after maximum), and E (end of decay). Errors are propagated statistical errors of the pulse profiles. Only the minus solution is shown, which is the physically reasonable solution (see Sect. 3.1.1). The plus solution can be obtained by transforming $\theta_{\mathrm{obs}}$ to $\pi-\theta_{\mathrm{obs}}$.

of energy bands and luminosity, as is suggested in the plus solution. Also, the maximum at $\theta_{\mathrm{obs}} \approx 40^{\circ}$ in the minus solution observed in the softer bands indicates halo emission at the base of the accretion stream, which one expects for lower $\theta_{\text {obs }}$ instead of values like $\theta_{\mathrm{obs}}>100^{\circ}$ in the plus solution. Therefore, it is difficult to describe the plus solution in a physical sense and we conclude that the minus solution is more appropriate for the emission of $4 \mathrm{U} 0115+63$. Our remaining study is performed using the minus solution.

\subsubsection{Intrinsic beam patterns and geometry}

The radiation seen by a distant observer has suffered relativistic light deflection. To understand the local emission pattern we imagined the radiation to originate from a point source at the pole and determined the intrinsic beam patterns of this hypothetical point source by relating the local direction of photon emission with the direction of propagation far away from the neutron star. To obtain these intrinsic beam patterns, we needed to make assumptions about the neutron star and remove the effects of the relativistic light deflection. We assumed a standard neutron star with $R_{\mathrm{NS}}=10 \mathrm{~km}$ and $M_{\mathrm{NS}}=1.4 M_{\odot}$. In Fig. 10 we plotted polar diagrams of the intrinsic beam patterns during the maximum and at the end of the decay. We only show the softest and the hardest bands for clarity.

The reconstruction of the total beam pattern provides us with two contraints on the pulsar geometry:

$a=\frac{\cot \Theta_{0}\left(\cos \Theta_{2}-\cos \Theta_{1}\right)}{\sin \Theta_{1}}, \quad b=\frac{\sin \Theta_{2}}{\sin \Theta_{1}}$

(see Kraus et al. 1995). The inclination angle $\Theta_{0}$ of the observer with respect to the rotation axis of the neutron star is a crucial number for the analysis. The $\Theta_{0}$-dependence of the position angles of the magnetic poles $\Theta_{1}$ and $\Theta_{2}$ and the offset $\delta$ from the antipodal configuration of the magnetic poles is shown in Fig. 11. The errors in Fig. 11 represent the uncertainty of the determination of $a$ and $b$ for the reconstruction of the beam patterns, 

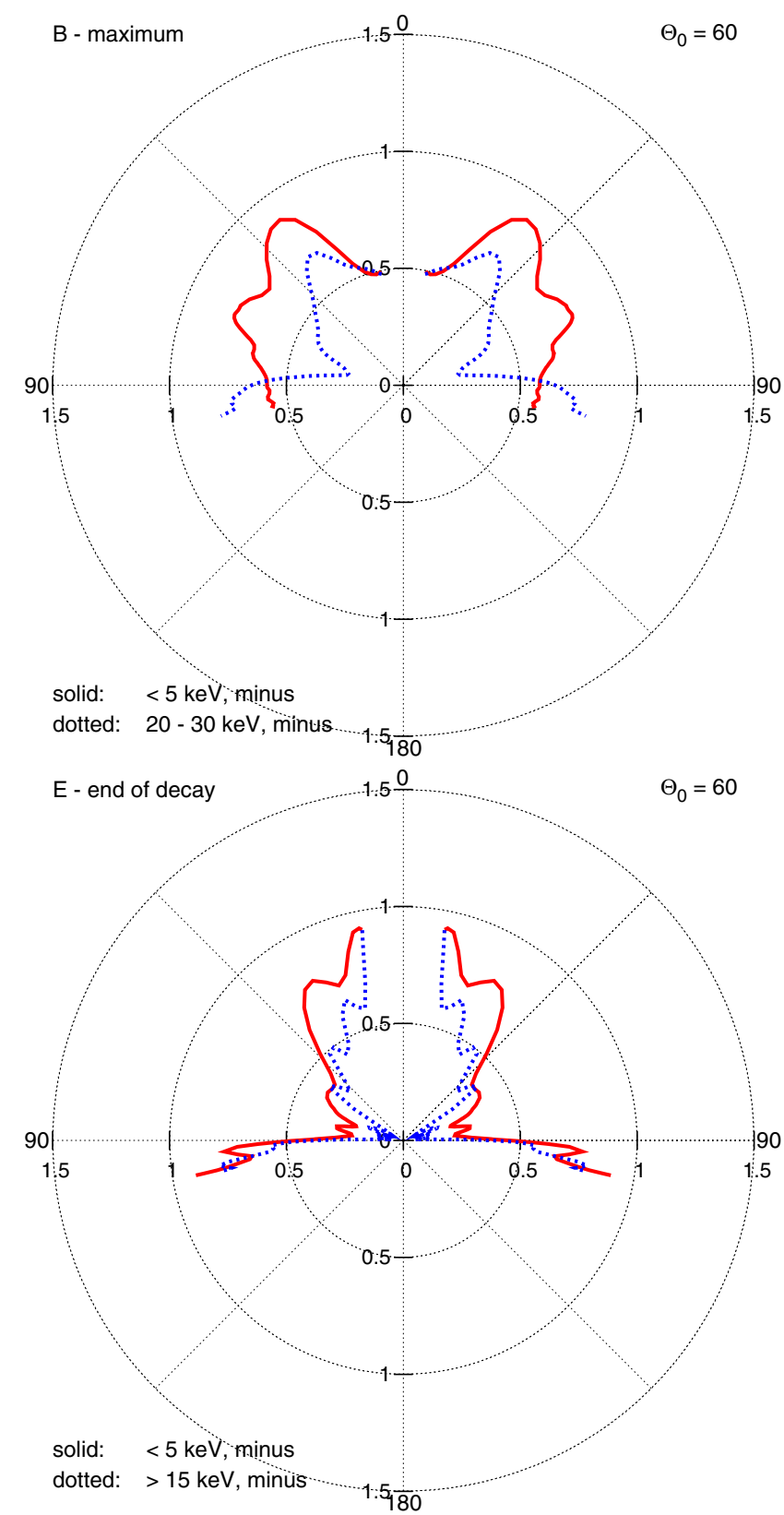

Fig. 10. Polar diagrams of the intrinsic beam patterns of $4 \mathrm{U} 0115+63$ for the observations B (maximum) and $\mathrm{E}$ (end of decay) for the softest band and the hard band above $\sim 20 \mathrm{keV}$. Up $\left(0^{\circ}\right)$ is the direction straight up from the magnetic pole, $90^{\circ}$ and $270^{\circ}$ are the directions tangential to the neutron star surface at the magnetic pole. These beam patterns correspond to the asymptotic beam patterns of the minus solution shown in Fig. 9.

which was made simultaneously for all analysed pulse profiles. If we determine $a$ and $b$ separately for each pulse profiles, the values lie within the shown errors (see Sect.3.1.1). For an assumed $\Theta_{0}=60^{\circ}$, we obtain $\Theta_{1}=148^{\circ}\left(+12^{\circ},-57^{\circ}\right), \Theta_{2}=$ $74^{\circ}\left(+31^{\circ},-44^{\circ}\right)$, and $\delta=65^{\circ}\left(+14^{\circ},-26^{\circ}\right)$.

\section{2. $\vee 0332+53$}

The pulse profiles of $\mathrm{V} 0332+53$ were decomposed in the same way as those of $4 \mathrm{U} 0115+63$. After rejecting decompositions based on the criteria listed at the beginning of this section, we obtained a region in the parameter space of $\left(\Phi_{1}, \Delta\right)=\left(91^{\circ}-110^{\circ}\right.$,

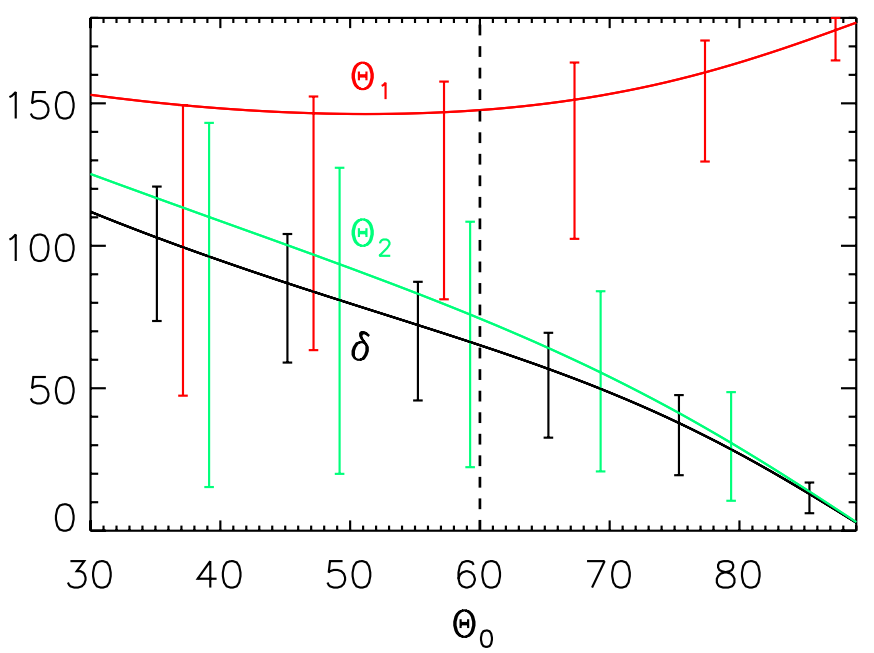

Fig. 11. Relation between the observing angle $\Theta_{0}$ and the angles $\Theta_{1}$ (red), $\Theta_{2}$ (green), and $\delta$ (black) for $4 \mathrm{U} 0115+63$. The vertical dashed line indicates $\Theta_{0}=60^{\circ}$. For errors see Sect. 3.1.2.

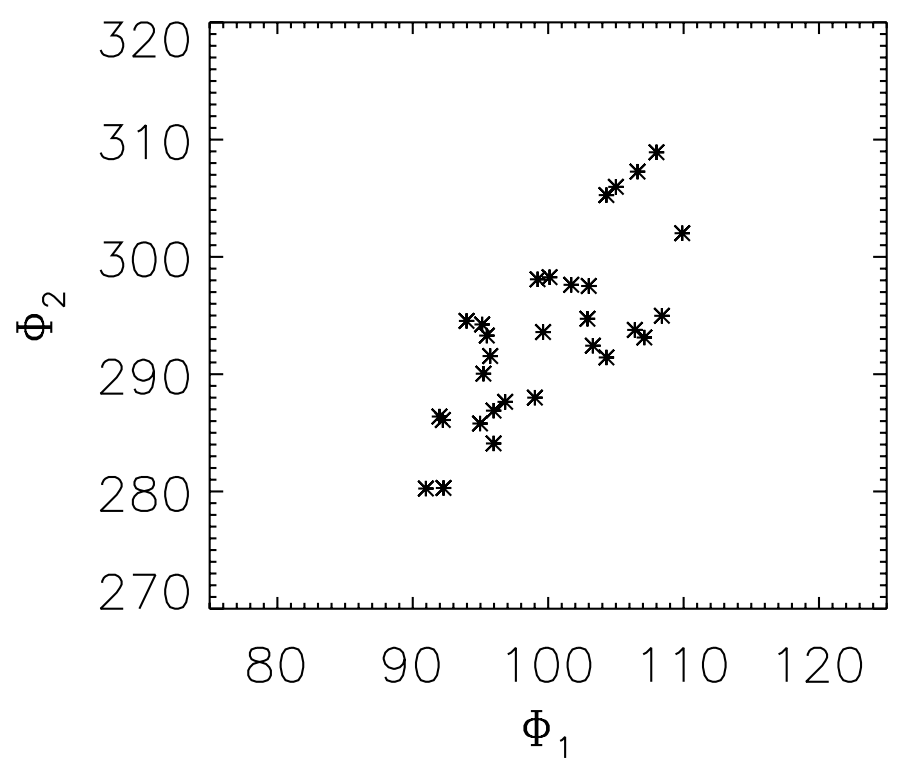

Fig. 12. Diagram showing the values of $\Phi_{1}$ and $\Phi_{2}$ for each pulse profile for the solution of V $0332+53$.

$6^{\circ}-21^{\circ}$ ). The resulting values for $\Phi_{1}$ and $\Phi_{2}$ for each pulse profile are plotted in Fig. 12. The parameters used for the reconstruction of the beam patterns are $a=-0.95(+0.35,-1.05)$, $b=1.0(+0.5,-0.5)$. Because there are no measurements of the inclination angle of $\mathrm{V} 0332+53$, we needed to assume an angle for the next steps of the analysis. The important features in the beam patterns (e.g., prominent maximum or minimum, increase towards lower or higher observation angles) do not change if we use different $\Theta_{0}$. Only the range of the angle for the intrinsic beam patterns discussed in Sect. 3.2.2 vary slightly with assumed $\Theta_{0}$. We chose to use an inclination angle of $\Theta_{0}=70^{\circ}$.

\subsubsection{Asymptotic beam patterns}

The single-pole pulse profiles of three pulse profiles of $\mathrm{V} 0332+53$ are shown in Fig. 13. Similar to the case of $4 \mathrm{U} 0115+63$ discussed in Sect. 3.1.1, the minus solution is reasonable for $\mathrm{V} 0332+53$. If we look at the asymptotic beam 

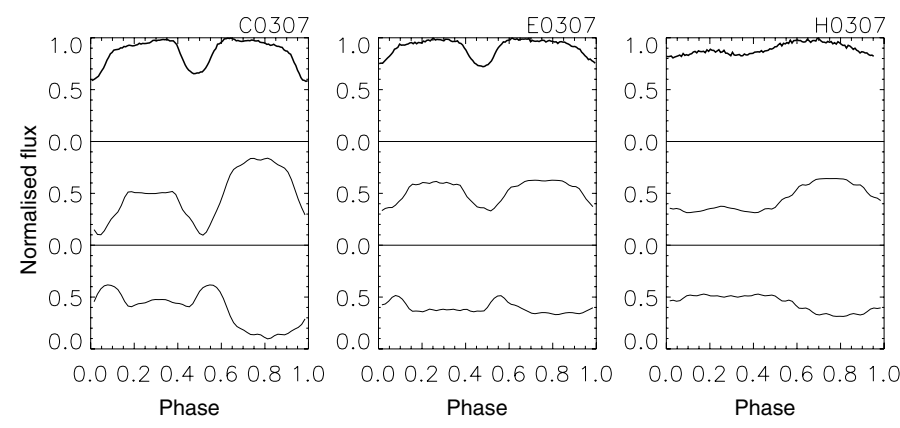

Fig. 13. Single-pole pulse profiles of V $0332+53$ for one pole (lower curve) and the other (middle) for the observations $\mathrm{C}$ (before maximum), $\mathrm{E}$ (after maximum), and $\mathrm{H}$ (end of decay) in the softest band. The upper thick curve shows the total pulse profile, which is the sum of the two single-pole pulse profiles.

patterns of the minus solution in Fig. 14, the main emission is found for low $\theta_{\mathrm{obs}}$ if the source is faint (A0307 - A2030 at the beginning and $\mathrm{H} 0307, \mathrm{H} 0720$ at the end of the giant outburst). If the source becomes brighter we find additional components for $\theta_{\mathrm{obs}} \approx 50-90^{\circ}$ and distinct minima at $\sim 50^{\circ}$ and $110^{\circ}$. These shapes of beam patterns can be adequately described using accretion column models (see below).

\subsubsection{Intrinsic beam patterns and geometry}

Intrinsic beam patterns were calculated assuming canonical values for the neutron star $\left(R_{\mathrm{NS}}=10 \mathrm{~km}\right.$ and $\left.M_{\mathrm{NS}}=1.4 M_{\odot}\right)$ and an observing angle of $\Theta_{0}=70^{\circ}$. The beam patterns are shown in Fig. 15. Clearly, at the beginning of the giant outburst, the emission of $\mathrm{V} 0332+53$ is mostly directed along the magnetic axis, indicating a pencil beam-like emission (Fig. 15, upper diagram).

Again, these results depend on the assumed observing angle $\Theta_{0}$. The position angles of the magnetic poles of $\mathrm{V} 0332+53$ are plotted over $\Theta_{0}$ in Fig. 16. For an assumed angle of $\Theta_{0}=70^{\circ}$, we obtain $\Theta_{1}=142^{\circ}\left(+18^{\circ},-22^{\circ}\right), \Theta_{2}=38^{\circ}\left(+8^{\circ},-12^{\circ}\right)$, and $\delta=12^{\circ}\left(+24^{\circ},-1^{\circ}\right)$.

\section{Discussion}

Kraus et al. (2003, in prep.) have performed model calculations of beam patterns and pulse profiles of X-ray pulsars in a luminosity range in which a radiative shock forms in the accretion column. The authors showed that in general three main components contribute to the observed flux: the first component is radiation observed directly from the accretion column. It forms a fan beam that is bent backwards towards the observer by relativistic light deflection while the corresponding pole and thus the accretion column is located on the back side of the neutron star. For standard neutron star parameters $\left(R_{\mathrm{NS}}=10 \mathrm{~km}\right.$ and $M_{\mathrm{NS}}=1.4 M_{\odot}$ ) the observed flux from the column peaks at $\theta_{\text {obs }}>140^{\circ}$. Secondly, the illumination of the neutron star surface creates a luminous halo that produces a broad pencil beam. Since the halo is shadowed when the accretion stream passes through the line of sight, the pencil beam is suppressed for low values of $\theta_{\text {obs }}$. The maximum of this "hollow pencil" is at $\theta_{\mathrm{obs}}=30-50^{\circ}$ for accretion funnel half widths of $5-10^{\circ}$ at the neutron star surface. For a hollow accretion column, there is an additional peak at $\theta_{\mathrm{obs}} \approx 0^{\circ}$ when the observer looks down into the hollow funnel. Thirdly, the upper accretion stream is irradiated by the column and the halo. Owing to advection in the accretion stream and gravitational light deflection, the reprocessed radiation forms a narrow anti-pencil, which is emission produced in the stream and is visible while the accreting pole is on the back side of the neutron star. The relative importance of these three components is energy-dependent; the halo is expected to dominate at low energies, the radiation reprocessed in the accretion stream is expected to dominate at the highest energies.

Cen X-3 was the first accreting X-ray pulsar to which the decomposition method was applied (Kraus et al. 1996) and for which a distorted geometry with a small offset $\delta$ was found. The derived beam patterns are a combination of a pencil and fan beam. Blum \& Kraus (2000) analysed Her X-1 and again found indications for a distorted magnetic field. They have also shown that in this source, a warped and tilted accretion disk attenuates the emission from one pole of the neutron star. In the case of A 0535+26 studied by Caballero et al. (2011), the most characteristic feature of the reconstructed beam pattern at all energies is the minimum in the flux at $\theta_{\text {obs }} \approx 30^{\circ}$. The authors performed model calculations to interpret the beam patterns for different parameters and found that a model including a hollow column emitting black body radiation plus a thermal halo created by radiation from the column walls, which was scattered on the neutron star surface, was able to reproduce the observations well. The results show that a hollow accretion column with an half-opening angle of $0.2 \mathrm{rad}\left(\sim 11^{\circ}\right)$ and column thickness of 0.06 rad with a halo around the base can well explain the deduced beam patterns. Based on the analysis of EXO 2030+375 we showed that a considerable fraction of its emission arises from a halo while the pole is facing the observer and from the accretion stream of the other pole while it is behind the neutron star (Sasaki et al. 2010). Even if the emission regions are behind the neutron star, gravitational light bending makes the emission visible to us.

The two sources analysed in this paper also fit in this scheme. The decompositions of the pulse profiles of the abovementioned accreting neutron stars indicate that the magnetic fields of these neutron stars are likely distorted to some extent. This is not so surprising because they might have experienced some anisotropic conditions during their birth depending on the environment in which the progenitor was located.

\section{1. $4 \cup 0115+63$}

We have already discussed in Sect. 3.1.1 that the minus solution is the likely solution for $4 \mathrm{U} 0115+63$. The comparison with the synthetic beam patterns of Kraus et al. (2003, in prep.) shows that the asymptotic and intrinsic beam patterns (Figs. 9 and 10, respectively) obtained at and near the maximum of the giant outburst (A and B data) can be interpreted in terms of an accretion stream with a column and a halo at the bottom. The halo component with a maximum at around $\theta_{\text {obs }}=50^{\circ}$ in the asymptotic beam patterns (Fig. 9) is most prominent in the softer bands around $10 \mathrm{keV}$ (the second and the third diagrams from the top in Fig. 9). This component fades above $\sim 15 \mathrm{keV}$ or if the luminosity drops to $\sim 10^{37} \mathrm{erg} \mathrm{s}^{-1}$. The emission from the accretion column is expected for higher $\theta_{\text {obs }}$. The halo and column components are also well reproduced for $\theta_{\text {intr }} \approx 30-90^{\circ}$ in the intrinsic beam patterns (Fig. 10, upper diagram). For $\theta_{\text {obs }} \approx 180^{\circ}$ (corresponding to $\theta_{\text {intr }} \approx 90^{\circ}$ ) there is a steep increase of the flux, which can be assigned to scattering in the accretion stream. This component is more prominent for higher energies $(>20 \mathrm{keV})$ and in particular in the end-of-decay data (Fig. 10, lower diagram).

The shape of the pulse profiles becomes simpler at the end of the giant outburst (E, F data), i.e., when the luminosity drops to about or less than the tenth of that during the maximum. The 

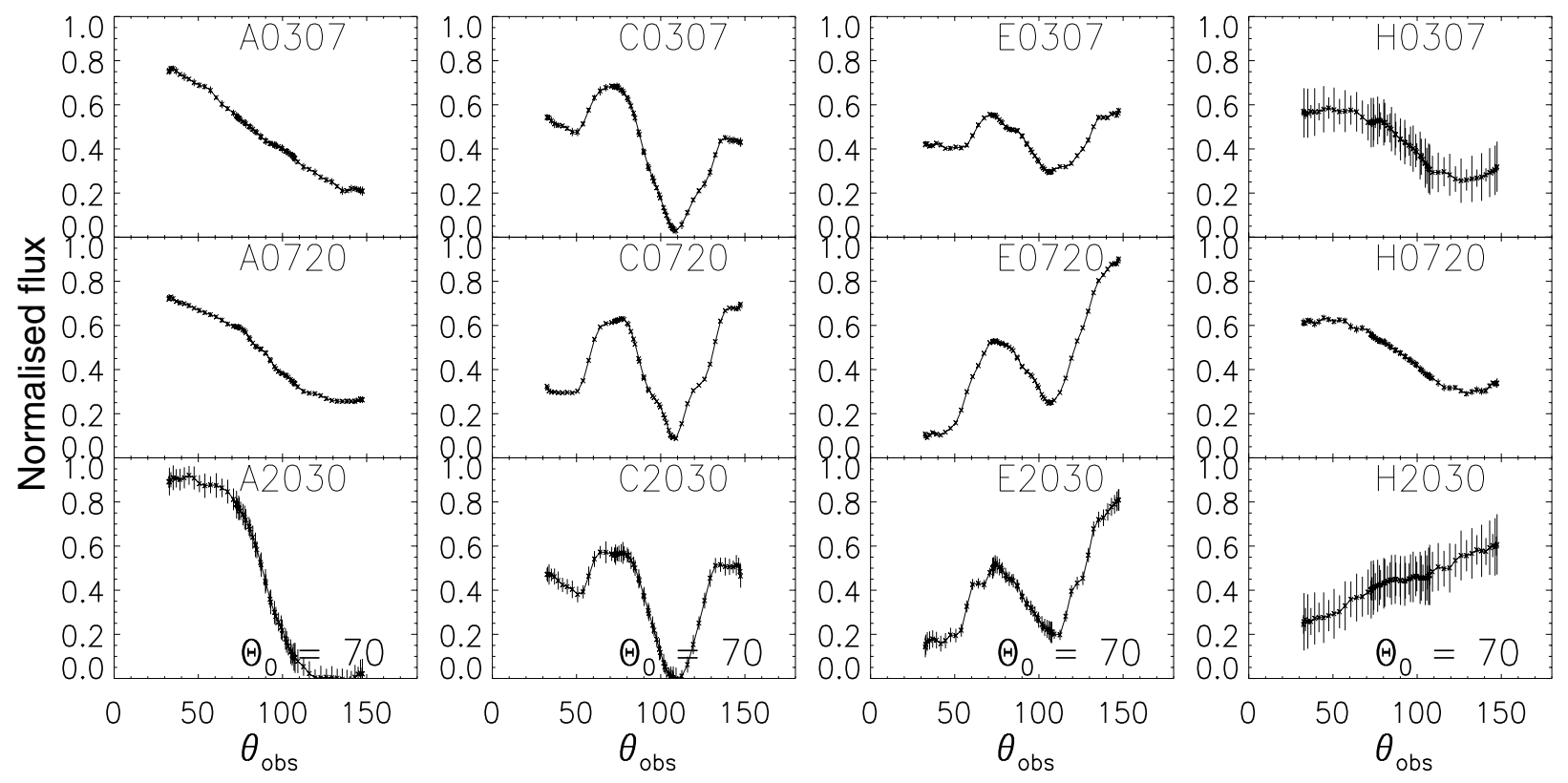

Fig. 14. Asymptotic beam patterns of V $0332+53$ for the observations A (rise), C (before maximum), E (after maximum), and $\mathrm{H}$ (end of decay). Errors are propagated statistical errors of the pulse profiles. Only the minus solution is shown (see Sect. 3.2).

beam patterns are simpler as well and show no extra maxima at $\theta_{\text {obs }} \approx 50$ or $100^{\circ}$. The accretion has decreased so much that the accretion column and also the halo have disappeared and we now see the emission from the hot spot as a pencil beam (Fig. 10, lower panel). This emission, however, can also be scattered in the upper accretion stream. Therefore, the rise for $\theta_{\mathrm{obs}}>120^{\circ}$ may be caused by the scattering in the accretion stream.

\section{2. $\vee 0332+53$}

The beam patterns obtained from the analysis of the pulse profiles of $\mathrm{V} 0332+53$ can also be interpreted in terms of an accretion column with halo emission and scattering in the upper accretion stream. The soft halo emission for $\theta_{\mathrm{obs}}<60^{\circ}$ is obvious in the data around the maximum $(\mathrm{C}, \mathrm{D}, \mathrm{E})$, while in these data, the scattering in the upper stream is prominent in the harder bands (observations $\mathrm{C}$ and $\mathrm{E}$ in Fig. 14). These components are also seen in the intrinsic beam patterns (Fig. 15, lower diagram), i.e., as halo emission for $\theta_{\text {intr }}<70^{\circ}$ and emission from the accretion stream for $\theta_{\text {intr }}>90^{\circ}$. In contrast to $4 \mathrm{U} 0115+63$, there is a pronounced minimum at $\theta_{\mathrm{obs}}=50^{\circ}$, which might indicate a hollow column. If more matter is funneled along the outer parts of the accretion column, the column can be optically thin in the center and regarded as a hollow column (Kraus 2001; Caballero et al. 2011). Because photons can also escape from the inner wall of the column, one sees emission for low $\theta_{\text {obs }}$. If the magnetic pole turns away from the observer, there is a point where the observer looks directly down on the top of the hollow column, i.e. on the shock front. No emission is observed directly from the column, like at the minimum at $\theta_{\mathrm{obs}}=50^{\circ}$. For even higher $\theta_{\text {obs }}$, the emission rises again because the observer now also sees emission from the outer wall of the accretion column.

However, shadowing of the halo emission by the upper stream can also cause reduced flux in the beam patterns. The emission that is directed perpendicular to the surface of the neutron star, and thus seen for lower $\theta_{\mathrm{obs}}$, can be most likely attributed to the halo. The fact that the minimum is more pronounced for higher energies than for lower energies (e.g., in the
E data) is in fact in favour of the shadowing of the halo emission because low-energy photons are scattered less than high-energy photons.

The most interesting result for $\mathrm{V} 0332+53$ is that we can directly observe the transition from a pencil-beam emission for low luminosities, hence low accretion rate (A), to a fan-beam emission from accretion column and scattered photons for higher luminosities $(\mathrm{C}, \mathrm{E})$, and again back to a pencil beam at the end of the giant outburst (H, see Figs. 14, 15). Only the shape of one beam pattern, i.e. that of $\mathrm{H} 2030$, is different from that of a pencil beam, but one should consider that the statistics for this data-set are very low (see Figs. 4, 5). This is the first observational proof for the accretion models that predict emission from a hot spot at the magnetic pole of the neutron star if the accretion rate is low, whereas an accretion column is formed for increased accretion rates.

\section{Conclusions}

We have studied the pulse profiles of the accreting X-ray pulsars 4U 0115+63 and V 0332+53 using BeppoSAX and RXTE data. For both sources, we analysed pulse profiles in different energy bands for many observations during one giant outburst to examine their energy and luminosity dependence. The pulse profiles were each decomposed into two symmetric single-pole pulse profiles. Using these two emission components we reconstructed the beam patterns of the X-ray pulsars for various energies and luminosity states.

The origin of the emission of $4 \mathrm{U} 0115+63$ during the highluminosity state can be interpreted as a combination of the accretion column, the halo at the bottom of the accretion column, and the scattering of photons in the upper accretion stream. The scattering in the upper accretion stream that causes the dominant emission for energies above $\sim 30 \mathrm{keV}$ also occurs for lower luminosities and creates the major peak in the observed total pulse profile. This emission is visible to the observer while the accretion stream is on the other side of the neutron star because of relativistic light bending. 


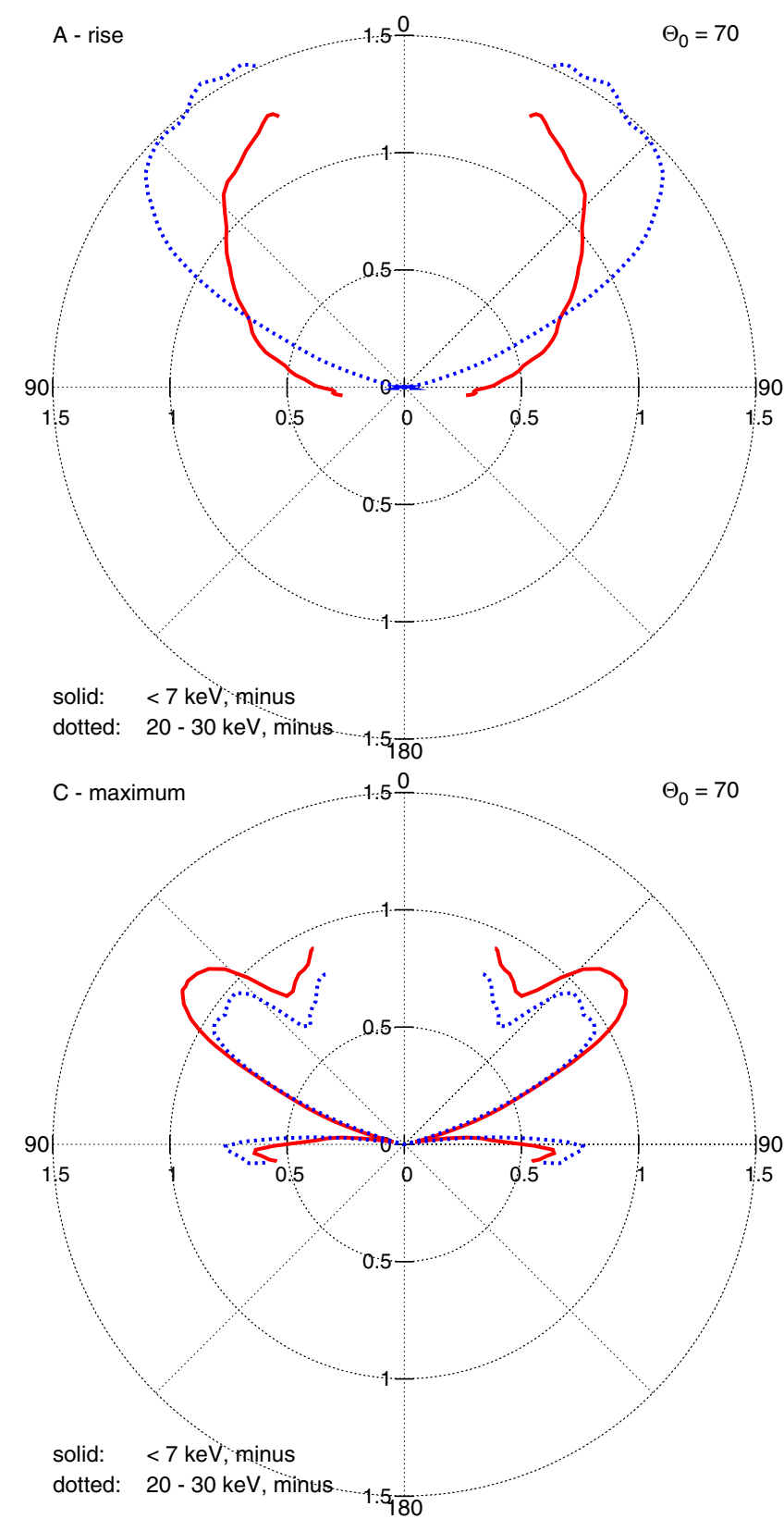

Fig. 15. Polar diagrams of the intrinsic beam patterns of $\mathrm{V} 0332+53$ for the observations $\mathrm{A}$ (rise) and $\mathrm{C}$ (maximum) for $<7 \mathrm{keV}$ and $20-30 \mathrm{keV}$, assuming an inclination angle of $\Theta_{0}=70^{\circ}$ between the line of sight and the rotation axis of the neutron star. Like in Fig. 10, up $\left(0^{\circ}\right)$ is the direction straight up from the magnetic pole, $90^{\circ}$ and $270^{\circ}$ are the directions tangential to the neutron star surface at the magnetic pole.

The analysis of the pulse profiles of $\mathrm{V} 0332+53$ showed that its beam patterns agree also with emission from the accretion column, the halo, and the upper accretion stream while the source is bright, and hence the accretion rate is high. For this source we found a minimum in the asymptotic beam patterns at $\theta_{\text {obs }}=50^{\circ}$, which is indicative of a hollow column or shadowing by the accretion stream.

The most important result for $\mathrm{V} 0332+53$ is that we found pencil-beam emission from the magnetic poles at the beginning and the end of the outburst, while other emission components are predominant during the outburst. By decomposing the observed pulse profiles into single-pole components, we showed for the first time that the change of the pulse profiles during the giant

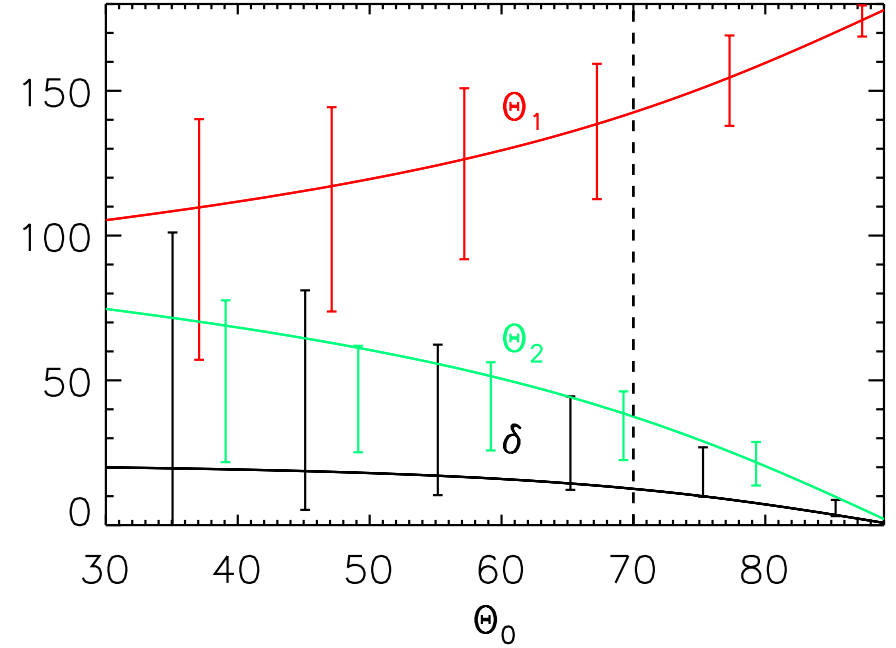

Fig. 16. Relation between the observing angle $\Theta_{0}$ and the angles $\Theta_{1}$ (red), $\Theta_{2}$ (green), and $\delta$ (black) for V $0332+53$. The vertical dashed line indicates $\Theta_{0}=70^{\circ}$. For errors see Sect.3.1.2.

outburst of $\mathrm{V} 0332+53$ is due to the transition from low accretion rate resulting in a mound, to higher accretion rate with the formation of a column and additional reprocessing on the neutron star surface as well as in the upper accretion stream, and back again to a mound when the accretion rate decreases.

Acknowledgements. This research has made use of data obtained from the High Energy Astrophysics Science Archive Research Center (HEASARC), provided by NASA's Goddard Space Flight Center. Quick-look results of the ASM are provided by the ASM/RXTE team. The BeppoSAX data have been provided by the Science data center of the Agenzia Spaziale Italiana (ASDC). This work was supported by DFG grant SA 1777 1/1.

\section{References}

Basko, M. M., \& Sunyaev, R. A. 1976, MNRAS, 175, 395

Baushev, A. N. 2009, Astron. Rep., 53, 67

Becker, P. A., \& Wolff, M. T. 2007, ApJ, 654, 435

Bernacca, P. L., Iijima, T., \& Stagni, R. 1984, A\&A, 132, L8

Bildsten, L., Chakrabarty, D., Chiu, J., et al. 1997, ApJS, 113, 367

Blum, S., \& Kraus, U. 2000, ApJ, 529, 968

Brainerd, J. J., \& Meszaros, P. 1991, ApJ, 369, 179

Bulik, T., Riffert, H., Meszaros, P., et al. 1995, ApJ, 444, 405

Caballero, I., Kraus, U., Santangelo, A., Sasaki, M., \& Kretschmar, P. 2011, A\&A, 526, A131

Coburn, W., Kretschmar, P., Kreykenbohm, I., et al. 2005, The Astronomer's Telegram, 381, 1

Cominsky, L., Clark, G. W., Li, F., Mayer, W., \& Rappaport, S. 1978, Nature, 273,367

Davidson, K. 1973, Nature, 246, 1

Davidson, K., \& Ostriker, J. P. 1973, ApJ, 179, 585

Ferrigno, C., Becker, P. A., Segreto, A., Mineo, T., \& Santangelo, A. 2009, A\&A, 498, 825

Forman, W., Tananbaum, H., \& Jones, C. 1976, ApJ, 206, L29

Frontera, F., \& Dalfiume, D. 1989, in Two Topics in X-Ray Astronomy, Vol. 1: X Ray Binaries, Vol. 2: AGN and the X Ray Background, ed. J. Hunt, \& B. Battrick, ESA SP, 296, 57

Hutchings, J. B., \& Crampton, D. 1981, ApJ, 247, 222

Inoue, H. 1985, Space Sci. Rev., 40, 317

Jahoda, K., Swank, J. H., Giles, A. B., et al. 1996, in SPIE Conf. Ser. 2808, ed. O. H. Siegmund, \& M. A. Gummin, 59

Johns, M., Koski, A., Canizares, C., et al. 1978, IAU Circ., 3171, 1

Johnston, M., Bradt, H., Doxsey, R., et al. 1978, ApJ, 223, L71

Kholopov, P. N., Samus', N. N., Kukarkina, N. P., Medvedeva, G. I., \& Perova, N. B. 1981, Info. Bull. Var. Stars, 2042, 1

Kouveliotou, C., van Paradijs, J., Fishman, G. J., et al. 1996, Nature, 379, 799

Kraus, U. 2001, ApJ, 563, 289

Kraus, U., Nollert, H.-P., Ruder, H., \& Riffert, H. 1995, ApJ, 450, 763

Kraus, U., Blum, S., Schulte, J., Ruder, H., \& Meszaros, P. 1996, ApJ, 467, 794 
Kraus, U., Zahn, C., Weth, C., \& Ruder, H. 2003, ApJ, 590, 424

Kreykenbohm, I., Mowlavi, N., Produit, N., et al. 2005, A\&A, 433, L45

Leahy, D. A. 1991, MNRAS, 251, 203

Lewin, W. H. G., Rutledge, R. E., Kommers, J. M., van Paradijs, J., \& Kouveliotou, C. 1996, ApJ, 462, L39

Lyubarskii, Y. E., \& Syunyaev, R. A. 1988, Sov. Astron. Lett., 14, 390

Miller, G. S. 1996, ApJ, 468, L29

Mowlavi, N., Kreykenbohm, I., Shaw, S. E., et al. 2006, A\&A, 451, 187

Nagase, F., Corbet, R. H. D., Day, C. S. R., et al. 1992, ApJ, 396, 147

Nakajima, M., Mihara, T., Makishima, K., \& Niko, H. 2006, ApJ, 646, 1125

Negueruela, I., \& Okazaki, A. T. 2001, A\&A, 369, 108

Negueruela, I., Roche, P., Fabregat, J., \& Coe, M. J. 1999, MNRAS, 307, 695

Parmar, A. N., White, N. E., \& Stella, L. 1989, ApJ, 338, 373

Pottschmidt, K., Kreykenbohm, I., Wilms, J., et al. 2005, ApJ, 634, L97

Rappaport, S., Clark, G. W., Cominsky, L., Li, F., \& Joss, P. C. 1978, ApJ, 224, L1
Riffert, H., \& Meszaros, P. 1988, ApJ, 325, 207

Riffert, H., Nollert, H.-P., Kraus, U., \& Ruder, H. 1993, ApJ, 406, 185

Santangelo, A., Segreto, A., Giarrusso, S., et al. 1999, ApJ, 523, L85

Sasaki, M., Klochkov, D., Kraus, U., Caballero, I., \& Santangelo, A. 2010, A\&A, 517, A8

Staubert, R., Kendziorra, E., Pietsch, W., et al. 1980, ApJ, 239, 1010

Stella, L., White, N. E., Davelaar, J., et al. 1985, ApJ, 288, L45

Terrell, J., \& Priedhorsky, W. C. 1984, ApJ, 285, L15

Tsygankov, S. S., Lutovinov, A. A., Churazov, E. M., \& Sunyaev, R. A. 2006, MNRAS, 371, 19

Tsygankov, S. S., Lutovinov, A. A., Churazov, E. M., \& Sunyaev, R. A. 2007, Astron. Lett., 33, 368

Wang, Y., \& Welter, G. L. 1981, A\&A, 102, 97

White, N. E., Swank, J. H., \& Holt, S. S. 1983, ApJ, 270, 711

Whitlock, L., Roussel-Dupre, D., \& Priedhorsky, W. 1989, ApJ, 338, 381

Zhang, S., Qu, J., Song, L., \& Torres, D. F. 2005, ApJ, 630, L65 